\title{
Some common fixed point results in ordered partial $b$-metric spaces
}

\author{
Zead Mustafa ${ }^{1}$, Jamal Rezaei Roshan ${ }^{2 *}$, Vahid Parvaneh ${ }^{3}$ and Zoran Kadelburg ${ }^{4}$
}

${ }^{*}$ Correspondence:

jmlroshan@gmail.com;

jml.roshan@qaemshahriau.ac.ir

${ }^{2}$ Department of Mathematics,

Qaemshahr Branch, Islamic Azad University, Qaemshahr, Iran

Full list of author information is

available at the end of the article

\begin{abstract}
In this paper, we introduce a modified version of ordered partial $b$-metric spaces. We demonstrate a fundamental lemma for the convergence of sequences in such spaces. Using this lemma, we prove some fixed point and common fixed point results for $(\psi, \varphi)$-weakly contractive mappings in the setup of ordered partial $b$-metric spaces. Finally, examples are presented to verify the effectiveness and applicability of our main results.
\end{abstract}

MSC: $47 \mathrm{H} 10 ; 54 \mathrm{H} 25$

Keywords: ordered metric space; partial metric space; $b$-metric space; common fixed point; altering distance function

\section{Introduction}

Fixed points theorems in partially ordered metric spaces were firstly obtained in 2004 by Ran and Reurings [1], and then by Nieto and Lopez [2]. In this direction several authors obtained further results under weak contractive conditions (see, e.g., [3-8]).

The concept of $b$-metric space was introduced by Bakhtin [9] and extensively used by Czerwik in $[10,11]$. After that, several interesting results about the existence of a fixed point for single-valued and multi-valued operators in (ordered) $b$-metric spaces have been obtained (see, e.g., [12-26]).

Definition 1 [10] Let $X$ be a (nonempty) set and $s \geq 1$ be a given real number. A function $d: X \times X \rightarrow \mathbb{R}^{+}$is a $b$-metric on $X$ if, for all $x, y, z \in X$, the following conditions hold:

(b $\left.\mathrm{b}_{1}\right) d(x, y)=0$ if and only if $x=y$,

$\left(\mathrm{b}_{2}\right) d(x, y)=d(y, x)$,

$\left(\mathrm{b}_{3}\right) d(x, z) \leq s[d(x, y)+d(y, z)]$.

In this case, the pair $(X, d)$ is called a $b$-metric space.

On the other hand, Matthews [27] introduced the notion of a partial metric space as a part of the study of denotational semantics of dataflow networks. In partial metric spaces, self-distance of an arbitrary point need not be equal to zero. Several authors obtained many useful fixed point results in these spaces - we mention just [28-33].

Definition 2 [27] A partial metric on a nonempty set $X$ is a mapping $p: X \times X \rightarrow \mathbb{R}^{+}$such that for all $x, y, z \in X$ :

@2013 Mustafa et al.; licensee Springer. This is an Open Access article distributed under the terms of the Creative Commons Attribution License (http://creativecommons.org/licenses/by/2.0), which permits unrestricted use, distribution, and reproduction in any medium, provided the original work is properly cited. 
$\left(\mathrm{p}_{1}\right) \quad x=y$ if and only if $p(x, x)=p(x, y)=p(y, y)$,

$\left(\mathrm{p}_{2}\right) p(x, x) \leq p(x, y)$

( $\left.\mathrm{p}_{3}\right) p(x, y)=p(y, x)$,

$\left(\mathrm{p}_{4}\right) \quad p(x, y) \leq p(x, z)+p(z, y)-p(z, z)$.

In this case, $(X, p)$ is called a partial metric space.

It is clear that if $p(x, y)=0$, then from $\left(\mathrm{p}_{1}\right)$ and $\left(\mathrm{p}_{2}\right), x=y$. But if $x=y, p(x, y)$ may not be 0 . A basic example of a partial metric space is the pair $\left(\mathbb{R}^{+}, p\right)$, where $p(x, y)=\max \{x, y\}$ for all $x, y \in \mathbb{R}^{+}$.

Each partial metric $p$ on a set $X$ generates a $T_{0}$ topology $\tau_{p}$ on $X$ which has as a base the family of open $p$-balls $\left\{B_{p}(x, \varepsilon): x \in X, \varepsilon>0\right\}$, where $B_{p}(x, \varepsilon)=\{y \in X: p(x, y)<p(x, x)+\varepsilon\}$ for all $x \in X$ and $\varepsilon>0$.

Definition 3 [27] Let $(X, p)$ be a partial metric space, and let $\left\{x_{n}\right\}$ be a sequence in $X$ and $x \in X$. Then:

(i) The sequence $\left\{x_{n}\right\}$ is said to converge to $x$ with respect to $\tau_{p}$ if $\lim _{n \rightarrow \infty} p\left(x_{n}, x\right)=$ $p(x, x)$.

(ii) The sequence $\left\{x_{n}\right\}$ is said to be Cauchy in $(X, p)$ if $\lim _{n, m \rightarrow \infty} p\left(x_{n}, x_{m}\right)$ exists and is finite.

(iii) $(X, p)$ is said to be complete if every Cauchy sequence $\left\{x_{n}\right\}$ in $X$ converges, with respect to $\tau_{p}$, to a point $x \in X$ such that $\lim _{n, m \rightarrow \infty} p\left(x_{n}, x_{m}\right)=\lim _{n \rightarrow \infty} p\left(x_{n}, x\right)=$ $p(x, x)$.

The following example shows that a convergent sequence $\left\{x_{n}\right\}$ in a partial metric space $(X, p)$ may not be Cauchy. In particular, it shows that the limit may not be unique.

Example 1 [32] Let $X=[0, \infty)$ and $p(x, y)=\max \{x, y\}$. Let

$$
x_{n}= \begin{cases}0, & n=2 k, \\ 1, & n=2 k+1 .\end{cases}
$$

Then, clearly, $\left\{x_{n}\right\}$ is a convergent sequence and for every $x \geq 1$, we have $\lim _{n \rightarrow \infty} p\left(x_{n}, x\right)=$ $p(x, x)$. But $\lim _{n, m \rightarrow \infty} p\left(x_{n}, x_{m}\right)$ does not exist, that is, $\left\{x_{n}\right\}$ is not a Cauchy sequence.

As a generalization and unification of partial metric and $b$-metric spaces, Shukla [34] introduced the concept of partial $b$-metric space as follows.

Definition 4 [34] A partial $b$-metric on a nonempty set $X$ is a mapping $p_{b}: X \times X \rightarrow \mathbb{R}^{+}$ such that for all $x, y, z \in X$ :

$\left(\mathrm{p}_{b 1}\right) \quad x=y$ if and only if $p_{b}(x, x)=p_{b}(x, y)=p_{b}(y, y)$,

$\left(\mathrm{p}_{b 2}\right) p_{b}(x, x) \leq p_{b}(x, y)$

$\left(\mathrm{p}_{b 3}\right) p_{b}(x, y)=p_{b}(y, x)$,

$\left(\mathrm{p}_{b 4}\right) p_{b}(x, y) \leq s\left[p_{b}(x, z)+p_{b}(z, y)\right]-p_{b}(z, z)$.

A partial $b$-metric space is a pair $\left(X, p_{b}\right)$ such that $X$ is a nonempty set and $p_{b}$ is a partial $b$-metric on $X$. The number $s \geq 1$ is called the coefficient of $\left(X, p_{b}\right)$. 
In a partial $b$-metric space $\left(X, p_{b}\right)$, if $x, y \in X$ and $p_{b}(x, y)=0$, then $x=y$, but the converse may not be true. It is clear that every partial metric space is a partial $b$-metric space with the coefficient $s=1$ and every $b$-metric space is a partial $b$-metric space with the same coefficient and zero self-distance. However, the converse of these facts need not hold.

Example 2 [34] Let $X=\mathbb{R}^{+}, q>1$ be a constant and $p_{b}: X \times X \rightarrow \mathbb{R}^{+}$be defined by

$$
p_{b}(x, y)=[\max \{x, y\}]^{q}+|x-y|^{q} \quad \text { for all } x, y \in X .
$$

Then $\left(X, p_{b}\right)$ is a partial $b$-metric space with the coefficient $s=2^{q-1}>1$, but it is neither a $b$-metric nor a partial metric space.

Note that in a partial $b$-metric space the limit of a convergent sequence may not be unique (see [34, Example 2]).

Some more examples of partial $b$-metrics can be constructed with the help of the following propositions.

Proposition 1 [34] Let $X$ be a nonempty set, and let $p$ be a partial metric and $d$ be a $b$-metric with the coefficient $s \geq 1$ on $X$. Then the function $p_{b}: X \times X \rightarrow \mathbb{R}^{+}$, defined by $p_{b}(x, y)=p(x, y)+d(x, y)$ for all $x, y \in X$, is a partial b-metric on $X$ with the coefficient $s$.

Proposition 2 [34] Let $(X, p)$ be a partial metric space and $q \geq 1$. Then $\left(X, p_{b}\right)$ is a partial $b$-metric space with the coefficient $s=2^{q-1}$, where $p_{b}$ is defined by $p_{b}(x, y)=[p(x, y)]^{q}$.

Altering distance functions were introduced by Khan et al. in [35].

Definition 5 [35] A function $\psi:[0, \infty) \rightarrow[0, \infty)$ is called an altering distance function if the following properties are satisfied:

1. $\psi$ is continuous and nondecreasing;

2. $\psi(t)=0$ if and only if $t=0$.

So far, many authors have studied fixed point theorems which are based on altering distance functions (see, e.g., [12, 28, 36-41]).

In this paper, we introduce a modified version of ordered partial $b$-metric spaces. We demonstrate a fundamental lemma for the convergence of sequences in such spaces. Using this lemma, we prove some fixed point and common fixed point results for $(\psi, \varphi)$-weakly contractive mappings in the setup of ordered partial $b$-metric spaces. Finally, examples are presented to verify the effectiveness and applicability of our main results.

\section{Definition and basic properties of partial $b$-metric spaces}

In the following definition, we modify Definition 4 in order to obtain that each partial $b$-metric $p_{b}$ generates a $b$-metric $d_{p_{b}}$.

Definition 6 Let $X$ be a (nonempty) set and $s \geq 1$ be a given real number. A function $p_{b}: X \times X \rightarrow \mathbb{R}^{+}$is a partial $b$-metric if, for all $x, y, z \in X$, the following conditions are satisfied:

$\left(\mathrm{p}_{b 1}\right) \quad x=y \Longleftrightarrow p_{b}(x, x)=p_{b}(x, y)=p_{b}(y, y)$,

$\left(\mathrm{p}_{b 2}\right) \quad p_{b}(x, x) \leq p_{b}(x, y)$, 
$\left(\mathrm{p}_{b 3}\right) \quad p_{b}(x, y)=p_{b}(y, x)$,

$\left(\mathrm{p}_{b 4^{\prime}}\right) p_{b}(x, y) \leq s\left(p_{b}(x, z)+p_{b}(z, y)-p_{b}(z, z)\right)+\left(\frac{1-s}{2}\right)\left(p_{b}(x, x)+p_{b}(y, y)\right)$.

The pair $\left(X, p_{b}\right)$ is called a partial $b$-metric space.

Since $s \geq 1$, from $\left(\mathrm{p}_{b 4^{\prime}}\right)$ we have

$$
p_{b}(x, y) \leq s\left(p_{b}(x, z)+p_{b}(z, y)-p_{b}(z, z)\right) \leq s\left(p_{b}(x, z)+p_{b}(z, y)\right)-p_{b}(z, z) .
$$

Hence, a partial $b$-metric in the sense of Definition 6 is also a partial $b$-metric in the sense of Definition 4.

It should be noted that the class of partial $b$-metric spaces is larger than the class of partial metric spaces, since a partial $b$-metric is a partial metric when $s=1$. We present an example which shows that a partial $b$-metric on $X$ (in the sense of Definition 6) might be neither a partial metric, nor a $b$-metric on $X$.

Example 3 Let $(X, d)$ be a metric space and $p_{b}(x, y)=d(x, y)^{q}+a$, where $q>1$ and $a \geq 0$ are real numbers. We will show that $p_{b}$ is a partial $b$-metric with $s=2^{q-1}$.

Obviously, conditions $\left(\mathrm{p}_{b 1}\right)-\left(\mathrm{p}_{b 3}\right)$ of Definition 6 are satisfied.

Since $q>1$, the convexity of the function $f(x)=x^{q}(x>0)$ implies that $(a+b)^{q} \leq 2^{q-1}\left(a^{q}+\right.$ $b^{q}$ ) holds for $a, b \geq 0$. Thus, for each $x, y, z \in X$, we obtain

$$
\begin{aligned}
p_{b}(x, y) & =d(x, y)^{q}+a \leq(d(x, z)+d(z, y))^{q}+a \\
& \leq 2^{q-1}\left(d(x, z)^{q}+d(z, y)^{q}\right)+a \\
& =2^{q-1}\left(d(x, z)^{q}+a+d(z, y)^{q}+a-a\right)+a-2^{q-1} a \\
& =2^{q-1}\left(p_{b}(x, z)+p_{b}(z, y)-p_{b}(z, z)\right)+\left(\frac{1-2^{q-1}}{2}\right)\left(p_{b}(x, x)+p_{b}(y, y)\right) .
\end{aligned}
$$

Hence, condition $\left(\mathrm{p}_{b 4^{\prime}}\right)$ of Definition 6 is fulfilled and $p_{b}$ is a partial $b$-metric on $X$.

Note that $\left(X, p_{b}\right)$ is not necessarily a partial metric space. For example, if $X=\mathbb{R}$ is the set of real numbers, $d(x, y)=|x-y|, q=2$ and $a=3$, then $p_{b}(x, y)=(x-y)^{2}+3$ is a partial $b$-metric on $X$ with $s=2^{2-1}=2$, but it is not a partial metric on $X$. Indeed, the ordinary (partial) triangle inequality does not hold. To see this, let $x=2, y=5$ and $z=\frac{5}{2}$. Then $p_{b}(2,5)=12, p_{b}\left(2, \frac{5}{2}\right)=\frac{13}{4}$ and $p_{b}\left(\frac{5}{2}, 5\right)=\frac{37}{4}$, hence $p_{b}(2,5)=12 \not \leq \frac{38}{4}=p_{b}\left(2, \frac{5}{2}\right)+p_{b}\left(\frac{5}{2}, 5\right)-$ $p_{b}\left(\frac{5}{2}, \frac{5}{2}\right)$.

Also, $p_{b}$ is not a $b$-metric since $p_{b}(x, x) \neq 0$ for $x \in X$.

Proposition 3 Every partial b-metric $p_{b}$ defines a b-metric $d_{p_{b}}$, where

$$
d_{p_{b}}(x, y)=2 p_{b}(x, y)-p_{b}(x, x)-p_{b}(y, y)
$$

for all $x, y \in X$.

Proof Let $x, y, z \in X$. Then we have

$$
\begin{aligned}
& d_{p_{b}}(x, y) \\
& \quad=2 p_{b}(x, y)-p_{b}(x, x)-p_{b}(y, y)
\end{aligned}
$$




$$
\begin{aligned}
\leq & 2\left[s\left(p_{b}(x, z)+p_{b}(z, y)-p_{b}(z, z)\right)+\left(\frac{1-s}{2}\right)\left(p_{b}(x, x)+p_{b}(y, y)\right)\right] \\
& -p_{b}(x, x)-p_{b}(y, y) \\
= & 2 s p_{b}(x, z)+2 s p_{b}(z, y)-2 s p_{b}(z, z)+(1-s)\left(p_{b}(x, x)+p_{b}(y, y)\right) \\
& -p_{b}(x, x)-p_{b}(y, y) \\
= & 2 s p_{b}(x, z)+2 s p_{b}(z, y)-2 s p_{b}(z, z)-s p_{b}(x, x)-s p_{b}(y, y) \\
= & s\left[2 p_{b}(x, z)-p_{b}(x, x)-p_{b}(z, z)+2 p_{b}(z, y)-p_{b}(z, z)-p_{b}(y, y)\right] \\
= & s\left[d_{p_{b}}(x, z)+d_{p_{b}}(z, y)\right] .
\end{aligned}
$$

Hence, the advantage of our definition of partial $b$-metric is that by using it we can define a dependent $b$-metric which we call the $b$-metric associated with $p_{b}$. This allows us to readily transport many concepts and results from $b$-metric spaces into a partial $b$-metric space.

Now, we present some definitions and propositions in a partial $b$-metric space.

Definition 7 Let $\left(X, p_{b}\right)$ be a partial $b$-metric space. Then, for $x \in X$ and $\epsilon>0$, the $p_{b}$-ball with center $x$ and radius $\epsilon$ is

$$
B_{p_{b}}(x, \epsilon)=\left\{y \in X \mid p_{b}(x, y)<p_{b}(x, x)+\epsilon\right\}
$$

For example, let $\left(X, p_{b}\right)$ be the partial $b$-metric space from Example 3 (with $X=\mathbb{R}, q=2$ and $a=3)$. Then

$$
\begin{aligned}
B_{p_{b}}(1,4) & =\left\{y \in X \mid p_{b}(1, y)<p_{b}(1,1)+4\right\}=\left\{y \in X \mid(y-1)^{2}+3<3+4\right\} \\
& =\left\{y \in X \mid(y-1)^{2}<4\right\}=(-1,3) .
\end{aligned}
$$

Proposition 4 Let $\left(X, p_{b}\right)$ be a partial b-metric space, $x \in X$ and $r>0$. If $y \in B_{p_{b}}(x, r)$, then there exists $\delta>0$ such that $B_{p_{b}}(y, \delta) \subseteq B_{p_{b}}(x, r)$.

Proof Let $y \in B_{p_{b}}(x, r)$. If $y=x$, then we choose $\delta=r$. Suppose that $y \neq x$. Then we have $p_{b}(x, y) \neq 0$. Now, we consider two cases.

Case 1. If $p_{b}(x, y)=p_{b}(x, x)$, then for $s=1$ we choose $\delta=r$. If $s>1$, then we consider the set

$$
A=\left\{n \in \mathbb{N} \mid \frac{r}{2 s^{n+1}(s-1)}<p_{b}(x, x)\right\}
$$

By the Archimedean property, $A$ is a nonempty set; then by the well ordering principle, $A$ has the least element $m$. Since $m-1 \notin A$, we have $p_{b}(x, x) \leq r /\left(2 s^{m}(s-1)\right)$ and we choose $\delta=r /\left(2 s^{m+1}\right)$. Let $z \in B_{p_{b}}(y, \delta)$; by the property $\left(\mathrm{p}_{b 4}\right)$, we have

$$
\begin{aligned}
p_{b}(x, z) & \leq s\left(p_{b}(x, y)+p_{b}(y, z)-p_{b}(y, y)\right) \\
& \leq s\left(p_{b}(x, x)+\delta\right) \\
& \leq p_{b}(x, x)+\frac{r}{2 s^{m}}+\frac{r}{2 s^{m}}
\end{aligned}
$$




$$
\begin{aligned}
& =p_{b}(x, x)+\frac{r}{s^{m}} \\
& <p_{b}(x, x)+r .
\end{aligned}
$$

Hence, $B_{p_{b}}(y, \delta) \subseteq B_{p_{b}}(x, r)$.

Case 2. If $p_{b}(x, y) \neq p_{b}(x, x)$, then from the property $\left(\mathrm{p}_{b 2}\right)$ we have $p_{b}(x, x)<p_{b}(x, y)$ and for $s=1$ we consider the set

$$
B=\left\{\begin{array}{l|l}
n \in \mathbb{N} & \frac{r}{2^{n+3}}<p_{b}(x, y)-p_{b}(x, x)
\end{array}\right\} .
$$

Similarly, by the well ordering principle, there exists an element $m$ such that $p_{b}(x, y)-$ $p_{b}(x, x) \leq r /\left(2^{m+2}\right)$, and we choose $\delta=r /\left(2^{m+2}\right)$. One can easily obtain that $B_{p_{b}}(y, \delta) \subseteq$ $B_{p_{b}}(x, r)$.

For $s>1$, we consider the set

$$
C=\left\{n \in \mathbb{N} \mid \frac{r}{2 s^{n+2}}<p_{b}(x, y)-\frac{1}{s} p_{b}(x, x)\right\}
$$

and by the well ordering principle, there exists an element $m$ such that $p_{b}(x, y)-\frac{1}{s} p_{b}(x, x) \leq$ $\frac{r}{2 s^{m+1}}$ and we choose $\delta=\frac{r}{2 s^{m+1}}$. Let $z \in B_{p_{b}}(y, \delta)$. By the property $\left(\mathrm{p}_{b 4}\right)$, we have

$$
\begin{aligned}
p_{b}(x, z) & \leq s\left(p_{b}(x, y)+p_{b}(y, z)-p_{b}(y, y)\right) \\
& \leq s\left(p_{b}(x, y)+\delta\right) \\
& \leq p_{b}(x, x)+\frac{r}{2 s^{m}}+\frac{r}{2 s^{m}} \\
& =p_{b}(x, x)+\frac{r}{s^{m}} \\
& <p_{b}(x, x)+r .
\end{aligned}
$$

Hence, $B_{p_{b}}(y, \delta) \subseteq B_{p_{b}}(x, r)$.

Thus, from the above proposition the family of all $p_{b}$-balls

$$
\Delta=\left\{B_{p_{b}}(x, r) \mid x \in X, r>0\right\}
$$

is a base of a $T_{0}$ topology $\tau_{p_{b}}$ on $X$ which we call the $p_{b}$-metric topology.

The topological space $\left(X, p_{b}\right)$ is $T_{0}$, but need not be $T_{1}$.

Definition 8 A sequence $\left\{x_{n}\right\}$ in a partial $b$-metric space $\left(X, p_{b}\right)$ is said to be:

(i) $p_{b}$-convergent to a point $x \in X$ if $\lim _{n \rightarrow \infty} p_{b}\left(x, x_{n}\right)=p_{b}(x, x)$;

(ii) a $p_{b}$-Cauchy sequence if $\lim _{n, m \rightarrow \infty} p_{b}\left(x_{n}, x_{m}\right)$ exists (and is finite).

(iii) A partial $b$-metric space $\left(X, p_{b}\right)$ is said to be $p_{b}$-complete if every $p_{b}$-Cauchy sequence $\left\{x_{n}\right\}$ in $X p_{b}$-converges to a point $x \in X$ such that $\lim _{n, m \rightarrow \infty} p_{b}\left(x_{n}, x_{m}\right)=$ $\lim _{n, m \rightarrow \infty} p_{b}\left(x_{n}, x\right)=p_{b}(x, x)$.

The following lemma shows the relationship between the concepts of $p_{b}$-convergence, $p_{b}$-Cauchyness and $p_{b}$-completeness in two spaces $\left(X, p_{b}\right)$ and $\left(X, d_{p_{b}}\right)$ which we state and prove according to Lemma 2.2 of [31]. 


\section{Lemma 1}

(1) A sequence $\left\{x_{n}\right\}$ is a $p_{b}$-Cauchy sequence in a partial $b$-metric space $\left(X, p_{b}\right)$ if and only if it is a $b$-Cauchy sequence in the $b$-metric space $\left(X, d_{p_{b}}\right)$.

(2) A partial $b$-metric space $\left(X, p_{b}\right)$ is $p_{b}$-complete if and only if the $b$-metric space

$\left(X, d_{p_{b}}\right)$ is $b$-complete. Moreover, $\lim _{n \rightarrow \infty} d_{p_{b}}\left(x, x_{n}\right)=0$ if and only if

$$
\lim _{n \rightarrow \infty} p_{b}\left(x, x_{n}\right)=\lim _{n, m \rightarrow \infty} p_{b}\left(x_{n}, x_{m}\right)=p_{b}(x, x) .
$$

Proof First, we show that every $p_{b}$-Cauchy sequence in $\left(X, p_{b}\right)$ is a $b$-Cauchy sequence in $\left(X, d_{p_{b}}\right)$. Let $\left\{x_{n}\right\}$ be a $p_{b}$-Cauchy sequence in $\left(X, p_{b}\right)$. Then, there exists $\alpha \in \mathbb{R}$ such that, for arbitrary $\varepsilon>0$, there is $n_{\varepsilon} \in \mathbb{N}$ with

$$
\left|p_{b}\left(x_{n}, x_{m}\right)-\alpha\right|<\frac{\varepsilon}{4}
$$

for all $n, m \geq n_{\varepsilon}$. Hence,

$$
\begin{aligned}
& \left|d_{p_{b}}\left(x_{n}, x_{m}\right)\right| \\
& \quad=2 p_{b}\left(x_{n}, x_{m}\right)-p_{b}\left(x_{n}, x_{n}\right)-p_{b}\left(x_{m}, x_{m}\right) \\
& \quad=\left|p_{b}\left(x_{n}, x_{m}\right)-\alpha+\alpha-p_{b}\left(x_{n}, x_{n}\right)+p_{b}\left(x_{m}, x_{n}\right)-\alpha+\alpha-p_{b}\left(x_{m}, x_{m}\right)\right| \\
& \quad \leq\left|p_{b}\left(x_{n}, x_{m}\right)-\alpha\right|+\left|\alpha-p_{b}\left(x_{n}, x_{n}\right)\right|+\left|p_{b}\left(x_{m}, x_{n}\right)-\alpha\right|+\left|\alpha-p_{b}\left(x_{m}, x_{m}\right)\right| \\
& \quad<\varepsilon
\end{aligned}
$$

for all $n, m \geq n_{\varepsilon}$. Hence, we conclude that $\left\{x_{n}\right\}$ is a $b$-Cauchy sequence in $\left(X, d_{p_{b}}\right)$.

Next, we prove that $b$-completeness of $\left(X, d_{p_{b}}\right)$ implies $p_{b}$-completeness of $\left(X, p_{b}\right)$. Indeed, if $\left\{x_{n}\right\}$ is a $p_{b}$-Cauchy sequence in $\left(X, p_{b}\right)$, then according to the above discussion, it is also a $b$-Cauchy sequence in $\left(X, d_{p_{b}}\right)$. Since the $b$-metric space $\left(X, d_{p_{b}}\right)$ is $b$-complete, we deduce that there exists $y \in X$ such that $\lim _{n \rightarrow \infty} d_{p_{b}}\left(y, x_{n}\right)=0$. Hence,

$$
\lim _{n \rightarrow \infty}\left[p_{b}\left(x_{n}, y\right)-p_{b}(y, y)+p_{b}\left(y, x_{n}\right)-p_{b}\left(x_{n}, x_{n}\right)\right]=0,
$$

therefore, $\lim _{n \rightarrow \infty}\left[p_{b}\left(x_{n}, y\right)-p_{b}(y, y)\right]=0$. Further, we have

$$
\lim _{n \rightarrow \infty}\left[p_{b}\left(y, x_{n}\right)-p_{b}\left(x_{n}, x_{n}\right)\right]=0 .
$$

Consequently,

$$
\lim _{n \rightarrow \infty} p_{b}\left(x_{n}, y\right)=p_{b}(y, y)=\lim _{n \rightarrow \infty} p_{b}\left(x_{n}, x_{n}\right) .
$$

On the other hand,

$$
\begin{aligned}
\lim _{n, m \rightarrow \infty} p_{b}\left(x_{n}, x_{m}\right) \leq & \lim _{n, m \rightarrow \infty} s p_{b}\left(x_{n}, y\right)+\lim _{n, m \rightarrow \infty} s p_{b}\left(x_{m}, y\right)-s p_{b}(y, y) \\
& +\left(\frac{1-s}{2}\right)\left(p_{b}\left(x_{n}, x_{n}\right)+p_{b}\left(x_{m}, x_{m}\right)\right) \\
= & p_{b}(y, y) .
\end{aligned}
$$


Also, from $\left(\mathrm{p}_{b 2}\right)$,

$$
p_{b}(y, y) \leq \lim _{n, m \rightarrow \infty} p_{b}\left(x_{n}, y\right)=\lim _{n, m \rightarrow \infty} p_{b}\left(x_{n}, x_{n}\right) \leq \lim _{n, m \rightarrow \infty} p_{b}\left(x_{n}, x_{m}\right)
$$

Hence, we obtain that $\left\{x_{n}\right\}$ is a $p_{b}$-convergent sequence in $\left(X, p_{b}\right)$.

Now, we prove that every $b$-Cauchy sequence $\left\{x_{n}\right\}$ in $\left(X, d_{p_{b}}\right)$ is a $p_{b}$-Cauchy sequence in $\left(X, p_{b}\right)$. Let $\varepsilon=\frac{1}{2}$. Then there exists $n_{0} \in \mathbb{N}$ such that $d_{p_{b}}\left(x_{n}, x_{m}\right)<\frac{1}{2}$ for all $n, m \geq n_{0}$. Since

$$
p_{b}\left(x_{n}, x_{n_{0}}\right)-p_{b}\left(x_{n_{0}}, x_{n_{0}}\right) \leq d_{p_{b}}\left(x_{n}, x_{n_{0}}\right)<\frac{1}{2},
$$

hence

$$
p_{b}\left(x_{n}, x_{n}\right) \leq p_{b}\left(x_{n}, x_{n_{0}}\right) \leq d_{p_{b}}\left(x_{n}, x_{n_{0}}\right)+p_{b}\left(x_{n_{0}}, x_{n_{0}}\right)<\frac{1}{2}+p_{b}\left(x_{n_{0}}, x_{n_{0}}\right) .
$$

Consequently, the sequence $\left\{p_{b}\left(x_{n}, x_{n}\right)\right\}$ is bounded in $\mathbb{R}$, and so there exists $a \in \mathbb{R}$ such that a subsequence $\left\{p_{b}\left(x_{n_{k}}, x_{n_{k}}\right)\right\}$ of $\left\{p_{b}\left(x_{n}, x_{n}\right)\right\}$ is convergent to $a$, i.e.,

$$
\lim _{k \rightarrow \infty} p_{b}\left(x_{n_{k}}, x_{n_{k}}\right)=a
$$

Now, we prove that $\left\{p_{b}\left(x_{n}, x_{n}\right)\right\}$ is a Cauchy sequence in $\mathbb{R}$. Since $\left\{x_{n}\right\}$ is a $b$-Cauchy sequence in $\left(X, d_{p_{b}}\right)$ for given $\varepsilon>0$, there exists $n_{\varepsilon} \in \mathbb{N}$ such that $d_{p_{b}}\left(x_{n}, x_{m}\right)<\varepsilon$ for all $n, m \geq n_{\varepsilon}$. Thus, for all $n, m \geq n_{\varepsilon}$,

$$
\begin{aligned}
p_{b}\left(x_{n}, x_{n}\right)-p_{b}\left(x_{m}, x_{m}\right) & \leq p_{b}\left(x_{n}, x_{m}\right)-p_{b}\left(x_{m}, x_{m}\right) \\
& \leq d_{p_{b}}\left(x_{m}, x_{n}\right)<\varepsilon .
\end{aligned}
$$

Therefore, $\lim _{n \rightarrow \infty} p_{b}\left(x_{n}, x_{n}\right)=a$.

On the other hand,

$$
\begin{aligned}
\left|p_{b}\left(x_{n}, x_{m}\right)-a\right| & =\left|p_{b}\left(x_{n}, x_{m}\right)-p_{b}\left(x_{n}, x_{n}\right)+p_{b}\left(x_{n}, x_{n}\right)-a\right| \\
& \leq d_{p_{b}}\left(x_{m}, x_{n}\right)+\left|p_{b}\left(x_{n}, x_{n}\right)-a\right|
\end{aligned}
$$

for all $n, m \geq n_{\varepsilon}$. Hence, $\lim _{n, m \rightarrow \infty} p_{b}\left(x_{n}, x_{m}\right)=a$, and consequently, $\left\{x_{n}\right\}$ is a $p_{b}$-Cauchy sequence in $\left(X, p_{b}\right)$.

Conversely, let $\left\{x_{n}\right\}$ be a $b$-Cauchy sequence in $\left(X, d_{p_{b}}\right)$. Then $\left\{x_{n}\right\}$ is a $p_{b}$-Cauchy sequence in $\left(X, p_{b}\right)$, and so it is convergent to a point $x \in X$ with

$$
\lim _{n \rightarrow \infty} p_{b}\left(x, x_{n}\right)=\lim _{n, m \rightarrow \infty} p_{b}\left(x_{m}, x_{n}\right)=p_{b}(x, x) .
$$

Then, for given $\varepsilon>0$, there exists $n_{\varepsilon} \in \mathbb{N}$ such that

$$
p_{b}\left(x, x_{n}\right)-p_{b}(x, x)<\frac{\varepsilon}{4}
$$

and

$$
p_{b}\left(x_{n}, x_{n}\right)-p_{b}(x, x) \leq p_{b}\left(x_{m}, x_{n}\right)-p_{b}(x, x)<\frac{\varepsilon}{4} .
$$


Therefore,

$$
\begin{aligned}
\left|d_{p_{b}}\left(x_{n}, x\right)\right| & =\left|p_{b}\left(x_{n}, x\right)-p_{b}\left(x_{n}, x_{n}\right)+p_{b}\left(x_{n}, x\right)-p_{b}(x, x)\right| \\
& \leq\left|p_{b}\left(x_{n}, x\right)-p_{b}(x, x)\right|+\left|p_{b}(x, x)-p_{b}\left(x_{n}, x_{n}\right)\right|+\left|p_{b}\left(x_{n}, x\right)-p_{b}(x, x)\right| \\
& <\varepsilon,
\end{aligned}
$$

whenever $n \geq n_{\varepsilon}$. Therefore, $\left(X, d_{p_{b}}\right)$ is complete.

Finally, let $\lim _{n \rightarrow \infty} d_{p_{b}}\left(x_{n}, x\right)=0$. So,

$$
\lim _{n \rightarrow \infty}\left[p_{b}\left(x_{n}, x\right)-p_{b}\left(x_{n}, x_{n}\right)\right]+\lim _{n \rightarrow \infty}\left[p_{b}\left(x_{n}, x\right)-p_{b}(x, x)\right]=0 .
$$

On the other hand,

$$
\begin{aligned}
\lim _{n, m \rightarrow \infty} & {\left[p_{b}\left(x_{n}, x_{m}\right)-p_{b}(x, x)\right] } \\
\leq & \lim _{n \rightarrow \infty}\left[s p_{b}\left(x_{n}, x\right)+s p_{b}\left(x, x_{m}\right)-s p_{b}(x, x)\right. \\
& \left.+\left(\frac{1-s}{2}\right)\left(p_{b}\left(x_{n}, x_{n}\right)+p_{b}\left(x_{m}, x_{m}\right)\right)-p_{b}(x, x)\right] \\
= & 0 .
\end{aligned}
$$

Definition 9 Let $\left(X, p_{b}\right)$ and $\left(X^{\prime}, p_{b}^{\prime}\right)$ be two partial $b$-metric spaces, and let $f:\left(X, p_{b}\right) \rightarrow$ $\left(X^{\prime}, p_{b}^{\prime}\right)$ be a mapping. Then $f$ is said to be $p_{b}$-continuous at a point $a \in X$ if for a given $\varepsilon>0$, there exists $\delta>0$ such that $x \in X$ and $p_{b}(a, x)<\delta+p_{b}(a, a)$ imply that $p_{b}^{\prime}(f(a), f(x))<$ $\varepsilon+p_{b}^{\prime}(f(a), f(a))$. The mapping $f$ is $p_{b}$-continuous on $X$ if it is $p_{b}$-continuous at all $a \in X$.

Proposition 5 Let $\left(X, p_{b}\right)$ and $\left(X^{\prime}, p_{b}^{\prime}\right)$ be two partial b-metric spaces. Then a mapping $f: X \rightarrow X^{\prime}$ is $p_{b}$-continuous at a point $x \in X$ if and only if it is $p_{b}$-sequentially continuous at $x$; that is, whenever $\left\{x_{n}\right\}$ is $p_{b}$-convergent to $x,\left\{f\left(x_{n}\right)\right\}$ is $p_{b}^{\prime}$-convergent to $f(x)$.

Definition 10 A triple $\left(X, \preceq, p_{b}\right)$ is called an ordered partial $b$-metric space if $(X, \preceq)$ is a partially ordered set and $p_{b}$ is a partial $b$-metric on $X$.

\section{Fixed point results in partial $b$-metric spaces}

The following crucial lemma is useful in proving our main results.

Lemma 2 Let $\left(X, p_{b}\right)$ be a partial b-metric space with the coefficient $s>1$ and suppose that $\left\{x_{n}\right\}$ and $\left\{y_{n}\right\}$ are convergent to $x$ and $y$, respectively. Then we have

$$
\begin{aligned}
\frac{1}{s^{2}} p_{b}(x, y)-\frac{1}{s} p_{b}(x, x)-p_{b}(y, y) & \leq \liminf _{n \rightarrow \infty} p_{b}\left(x_{n}, y_{n}\right) \leq \limsup _{n \rightarrow \infty} p_{b}\left(x_{n}, y_{n}\right) \\
& \leq s p_{b}(x, x)+s^{2} p_{b}(y, y)+s^{2} p_{b}(x, y) .
\end{aligned}
$$

In particular, if $p_{b}(x, y)=0$, then we have $\lim _{n \rightarrow \infty} p_{b}\left(x_{n}, y_{n}\right)=0$. 
Moreover, for each $z \in X$, we have

$$
\begin{aligned}
\frac{1}{s} p_{b}(x, z)-p_{b}(x, x) & \leq \liminf _{n \rightarrow \infty} p_{b}\left(x_{n}, z\right) \leq \limsup _{n \rightarrow \infty} p_{b}\left(x_{n}, z\right) \\
& \leq s p_{b}(x, z)+s p_{b}(x, x) .
\end{aligned}
$$

In particular, if $p_{b}(x, x)=0$, then we have

$$
\frac{1}{s} p_{b}(x, z) \leq \liminf _{n \rightarrow \infty} p_{b}\left(x_{n}, z\right) \leq \limsup _{n \rightarrow \infty} p_{b}\left(x_{n}, z\right) \leq s p_{b}(x, z)
$$

Proof Using the triangle inequality in a partial $b$-metric space, it is easy to see that

$$
p_{b}(x, y) \leq s p_{b}\left(x, x_{n}\right)+s^{2} p_{b}\left(x_{n}, y_{n}\right)+s^{2} p_{b}\left(y_{n}, y\right)
$$

and

$$
p_{b}\left(x_{n}, y_{n}\right) \leq s p_{b}\left(x_{n}, x\right)+s^{2} p_{b}(x, y)+s^{2} p_{b}\left(y, y_{n}\right) .
$$

Taking the lower limit as $n \rightarrow \infty$ in the first inequality and the upper limit as $n \rightarrow \infty$ in the second inequality, we obtain the first desired result. If $p_{b}(x, y)=0$, then by the triangle inequality we get $p_{b}(x, x)=0$ and $p_{b}(y, y)=0$. Therefore, we have $\lim _{n \rightarrow \infty} p_{b}\left(x_{n}, y_{n}\right)=0$. Similarly, using again the triangle inequality, the other assertions follow.

Let $\left(X, \preceq, p_{b}\right)$ be an ordered partial $b$-metric space, and let $f: X \rightarrow X$ be a mapping. Set

$$
M_{s}^{f}(x, y)=\max \left\{p_{b}(x, y), p_{b}(x, f x), p_{b}(y, f y), \frac{p_{b}(x, f y)+p_{b}(y, f x)}{2 s}\right\} .
$$

Definition 11 Let $\left(X, p_{b}\right)$ be an ordered partial $b$-metric space. We say that a mapping $f: X \rightarrow X$ is a generalized $(\psi, \varphi)_{s}$-weakly contractive mapping if there exist two altering distance functions $\psi$ and $\varphi$ such that

$$
\psi\left(s p_{b}(f x, f y)\right) \leq \psi\left(M_{s}^{f}(x, y)\right)-\varphi\left(M_{s}^{f}(x, y)\right)
$$

for all comparable $x, y \in X$.

First, we prove the following result.

Theorem 1 Let $\left(X, \preceq, p_{b}\right)$ be a $p_{b}$-complete ordered partial b-metric space. Let $f: X \rightarrow X$ be a nondecreasing, with respect to $\preceq$, continuous mapping. Suppose that $f$ is a generalized $(\psi, \varphi)_{s}$-weakly contractive mapping. If there exists $x_{0} \in X$ such that $x_{0} \preceq f x_{0}$, then $f$ has a fixed point.

Proof Let $x_{0} \in X$ be such that $x_{0} \preceq f x_{0}$. Then we define a sequence $\left(x_{n}\right)$ in $X$ such that $x_{n+1}=f x_{n}$ for all $n \geq 0$. Since $x_{0} \preceq f x_{0}=x_{1}$ and $f$ is nondecreasing, we have $x_{1}=f x_{0} \preceq x_{2}=$ 
$f x_{1}$. Again, as $x_{1} \preceq x_{2}$ and $f$ is nondecreasing, we have $x_{2}=f x_{1} \preceq x_{3}=f x_{2}$. By induction, we have

$$
x_{0} \preceq x_{1} \preceq \cdots \preceq x_{n} \preceq x_{n+1} \preceq \cdots .
$$

If $x_{n}=x_{n+1}$ for some $n \in \mathbb{N}$, then $x_{n}=f x_{n}$ and hence $x_{n}$ is a fixed point of $f$. So, we may assume that $x_{n} \neq x_{n+1}$ for all $n \in \mathbb{N}$. By (3.1), we have

$$
\begin{aligned}
\psi\left(p_{b}\left(x_{n}, x_{n+1}\right)\right) & \leq \psi\left(\operatorname{sp}_{b}\left(x_{n}, x_{n+1}\right)\right) \\
& =\psi\left(s_{b}\left(f x_{n-1}, f x_{n}\right)\right) \\
& \leq \psi\left(M_{s}^{f}\left(x_{n-1}, x_{n}\right)\right)-\varphi\left(M_{s}^{f}\left(x_{n-1}, x_{n}\right)\right)
\end{aligned}
$$

where

$$
\begin{aligned}
M_{s}^{f}\left(x_{n-1}, x_{n}\right)= & \max \left\{p_{b}\left(x_{n-1}, x_{n}\right), p_{b}\left(x_{n-1}, f x_{n-1}\right), p_{b}\left(x_{n}, f x_{n}\right),\right. \\
& \left.\frac{p_{b}\left(x_{n-1}, f x_{n}\right)+p_{b}\left(x_{n}, f x_{n-1}\right)}{2 s}\right\} \\
= & \max \left\{p_{b}\left(x_{n-1}, x_{n}\right), p_{b}\left(x_{n}, x_{n+1}\right), \frac{p_{b}\left(x_{n-1}, x_{n+1}\right)+p_{b}\left(x_{n}, x_{n}\right)}{2 s}\right\} \\
\leq & \max \left\{p_{b}\left(x_{n-1}, x_{n}\right), p_{b}\left(x_{n}, x_{n+1}\right),\right. \\
& \left.\frac{s p_{b}\left(x_{n-1}, x_{n}\right)+s p_{b}\left(x_{n}, x_{n+1}\right)+(1-s) p_{b}\left(x_{n}, x_{n}\right)}{2 s}\right\} \\
= & \max \left\{p_{b}\left(x_{n-1}, x_{n}\right), p_{b}\left(x_{n}, x_{n+1}\right)\right\} .
\end{aligned}
$$

So, we have

$$
M_{s}^{f}\left(x_{n-1}, x_{n}\right)=\max \left\{p_{b}\left(x_{n-1}, x_{n}\right), p_{b}\left(x_{n}, x_{n+1}\right)\right\} .
$$

From (3.2), (3.3) we get

$$
\begin{aligned}
\psi\left(p_{b}\left(x_{n}, x_{n+1}\right)\right) \leq & \psi\left(\max \left\{p_{b}\left(x_{n-1}, x_{n}\right), p_{b}\left(x_{n}, x_{n+1}\right)\right\}\right) \\
& -\varphi\left(\max \left\{p_{b}\left(x_{n-1}, x_{n}\right), p_{b}\left(x_{n}, x_{n+1}\right)\right\}\right) .
\end{aligned}
$$

If

$$
\max \left\{p_{b}\left(x_{n-1}, x_{n}\right), p_{b}\left(x_{n}, x_{n+1}\right)\right\}=p_{b}\left(x_{n}, x_{n+1}\right),
$$

then by (3.4) and properties of $\varphi$, we have

$$
\begin{aligned}
\psi\left(p_{b}\left(x_{n}, x_{n+1}\right)\right) & \leq \psi\left(p_{b}\left(x_{n}, x_{n+1}\right)\right)-\varphi\left(p_{b}\left(x_{n}, x_{n+1}\right)\right) \\
& <\psi\left(p_{b}\left(x_{n}, x_{n+1}\right)\right)
\end{aligned}
$$


which gives a contradiction. Thus,

$$
\psi\left(p_{b}\left(x_{n}, x_{n+1}\right)\right) \leq \psi\left(p_{b}\left(x_{n-1}, x_{n}\right)\right)-\varphi\left(p_{b}\left(x_{n-1}, x_{n}\right)\right)
$$

Therefore, $\left\{p_{b}\left(x_{n}, x_{n+1}\right): n \in \mathbb{N} \cup\{0\}\right\}$ is a nonincreasing sequence of positive numbers. So, there exists $r \geq 0$ such that

$$
\lim _{n \rightarrow \infty} p_{b}\left(x_{n}, x_{n+1}\right)=r
$$

Letting $n \rightarrow \infty$ in (3.5), we get

$$
\psi(r) \leq \psi(r)-\varphi(r) \leq \psi(r) .
$$

Therefore, $\varphi(r)=0$, and hence $r=0$. Thus, we have

$$
\lim _{n \rightarrow \infty} p_{b}\left(x_{n}, x_{n+1}\right)=0
$$

Next, we show that $\left\{x_{n}\right\}$ is a $p_{b}$-Cauchy sequence in $X$. For this, we have to show that $\left\{x_{n}\right\}$ is a $b$-Cauchy sequence in $\left(X, d_{p_{b}}\right)$ (see Lemma 1$)$. Suppose the contrary; that is, $\left\{x_{n}\right\}$ is not a $b$-Cauchy sequence. Then there exists $\varepsilon>0$ for which we can find two subsequences $\left\{x_{m_{i}}\right\}$ and $\left\{x_{n_{i}}\right\}$ of $\left\{x_{n}\right\}$ such that $n_{i}$ is the smallest index for which

$$
n_{i}>m_{i}>i, \quad d_{p_{b}}\left(x_{m_{i}}, x_{n_{i}}\right) \geq \varepsilon .
$$

This means that

$$
d_{p_{b}}\left(x_{m_{i}}, x_{n_{i}-1}\right)<\varepsilon
$$

From (3.7) and using the triangular inequality, we get

$$
\varepsilon \leq d_{p_{b}}\left(x_{m_{i}}, x_{n_{i}}\right) \leq s d_{p_{b}}\left(x_{m_{i}}, x_{n_{i}-1}\right)+s d_{p_{b}}\left(x_{n_{i}-1}, x_{n_{i}}\right) .
$$

Taking the upper limit as $i \rightarrow \infty$ and using (3.8), we get

$$
\frac{\varepsilon}{s} \leq \liminf _{i \rightarrow \infty} d_{p_{b}}\left(x_{m_{i}}, x_{n_{i}-1}\right) \leq \limsup _{i \rightarrow \infty} d_{p_{b}}\left(x_{m_{i}}, x_{n_{i}-1}\right) \leq \varepsilon
$$

Also, from (3.9) and (3.10),

$$
\varepsilon \leq \limsup _{i \rightarrow \infty} d_{p_{b}}\left(x_{m_{i}}, x_{n_{i}}\right) \leq s \varepsilon
$$

Further

$$
d_{p_{b}}\left(x_{m_{i}+1}, x_{n_{i}}\right) \leq s d_{p_{b}}\left(x_{m_{i}+1}, x_{m_{i}}\right)+s d_{p_{b}}\left(x_{m_{i}}, x_{n_{i}}\right),
$$

and hence

$$
\limsup _{i \rightarrow \infty} d_{p_{b}}\left(x_{m_{i}+1}, x_{n_{i}}\right) \leq s^{2} \varepsilon
$$


Finally,

$$
d_{p_{b}}\left(x_{m_{i}+1}, x_{n_{i}-1}\right) \leq s d_{p_{b}}\left(x_{m_{i}+1}, x_{m_{i}}\right)+s d_{p_{b}}\left(x_{m_{i}}, x_{n_{i}-1}\right)
$$

and hence

$$
\limsup _{i \rightarrow \infty} d_{p_{b}}\left(x_{m_{i}+1}, x_{n_{i}-1}\right) \leq s \varepsilon .
$$

On the other hand, by the definition of $d_{p_{b}}$ and (3.6),

$$
\limsup _{i \rightarrow \infty} d_{p_{b}}\left(x_{m_{i}}, x_{n_{i}-1}\right)=2 \underset{i \rightarrow \infty}{\limsup } p_{b}\left(x_{m_{i}}, x_{n_{i}-1}\right) .
$$

Hence, by (3.10),

$$
\frac{\varepsilon}{2 s} \leq \liminf _{i \rightarrow \infty} p_{b}\left(x_{m_{i}}, x_{n_{i}-1}\right) \leq \limsup _{i \rightarrow \infty} p_{b}\left(x_{m_{i}}, x_{n_{i}-1}\right) \leq \frac{\varepsilon}{2} .
$$

Similarly,

$$
\begin{aligned}
& \limsup _{i \rightarrow \infty} p_{b}\left(x_{m_{i}}, x_{n_{i}}\right) \leq \frac{s \varepsilon}{2}, \\
& \frac{\varepsilon}{2 s} \leq \limsup _{i \rightarrow \infty} p_{b}\left(x_{m_{i}+1}, x_{n_{i}}\right), \\
& \limsup _{i \rightarrow \infty} p_{b}\left(x_{m_{i}+1}, x_{n_{i}-1}\right) \leq \frac{s \varepsilon}{2} .
\end{aligned}
$$

From (3.1), we have

$$
\begin{aligned}
\psi\left(\operatorname{sp}_{b}\left(x_{m_{i}+1}, x_{n_{i}}\right)\right) & =\psi\left(\operatorname{sp}_{b}\left(f x_{m_{i}}, f x_{n_{i}-1}\right)\right) \\
& \leq \psi\left(M_{s}^{f}\left(x_{m_{i}}, x_{n_{i}-1}\right)\right)-\varphi\left(M_{s}^{f}\left(x_{m_{i}}, x_{n_{i}-1}\right)\right),
\end{aligned}
$$

where

$$
\begin{aligned}
M_{s}^{f}( & \left.x_{m_{i}}, x_{n_{i}-1}\right) \\
= & \max \left\{p_{b}\left(x_{m_{i}}, x_{n_{i}-1}\right), p_{b}\left(x_{m_{i}}, f x_{m_{i}}\right), p_{b}\left(x_{n_{i}-1}, f x_{n_{i}-1}\right),\right. \\
& \left.\frac{p_{b}\left(x_{m_{i}}, f x_{n_{i}-1}\right)+p_{b}\left(f x_{m_{i}}, x_{n_{i}-1}\right)}{2 s}\right\} \\
= & \max \left\{p_{b}\left(x_{m_{i}}, x_{n_{i}-1}\right), p_{b}\left(x_{m_{i}}, x_{m_{i}+1}\right), p_{b}\left(x_{n_{i}-1}, x_{n_{i}}\right),\right. \\
& \left.\frac{p_{b}\left(x_{m_{i}}, x_{n_{i}}\right)+p_{b}\left(x_{m_{i}+1}, x_{n_{i}-1}\right)}{2 s}\right\} .
\end{aligned}
$$


Taking the upper limit as $i \rightarrow \infty$ in (3.16) and using (3.6), (3.11), (3.12) and (3.14), we get

$$
\begin{aligned}
\limsup _{i \rightarrow \infty} M_{s}^{f}\left(x_{m_{i}}, x_{n_{i}-1}\right)= & \max \left\{\limsup _{i \rightarrow \infty} p_{b}\left(x_{m_{i}}, x_{n_{i}-1}\right), 0,0,\right. \\
& \left.\frac{\limsup \sup _{i \rightarrow \infty} p_{b}\left(x_{m_{i}}, x_{n_{i}}\right)+\lim \sup _{i \rightarrow \infty} p_{b}\left(x_{m_{i}+1}, x_{n_{i}-1}\right)}{2 s}\right\} \\
\leq & \max \left\{\frac{\varepsilon}{2}, \frac{\frac{\varepsilon s+\varepsilon s}{2}}{2 s}\right\}=\frac{\varepsilon}{2} .
\end{aligned}
$$

Now, taking the upper limit as $i \rightarrow \infty$ in (3.15) and using (3.13) and (3.17), we have

$$
\begin{aligned}
\psi\left(s \frac{\varepsilon}{2 s}\right) & \leq \psi\left(s \limsup _{i \rightarrow \infty} p_{b}\left(x_{m_{i}+1}, x_{n_{i}}\right)\right) \\
& \leq \psi\left(\limsup _{i \rightarrow \infty} M_{s}^{f}\left(x_{m_{i}}, x_{n_{i}-1}\right)\right)-\liminf _{i \rightarrow \infty} \varphi\left(M_{s}^{f}\left(x_{m_{i}}, x_{n_{i}-1}\right)\right) \\
& \leq \psi\left(\frac{\varepsilon}{2}\right)-\varphi\left(\liminf _{i \rightarrow \infty} M_{s}^{f}\left(x_{m_{i}}, x_{n_{i}-1}\right)\right),
\end{aligned}
$$

which further implies that

$$
\varphi\left(\liminf _{i \rightarrow \infty} M_{s}^{f}\left(x_{m_{i}}, x_{n_{i}-1}\right)\right)=0,
$$

so $\liminf _{i \rightarrow \infty} M_{s}^{f}\left(x_{m_{i}}, x_{n_{i}-1}\right)=0$, and by (3.16) we get $\liminf _{i \rightarrow \infty} d_{p_{b}}\left(x_{m_{i}}, x_{n_{i}-1}\right)=0$, a contradiction with (3.11).

Thus, we have proved that $\left\{x_{n}\right\}$ is a $b$-Cauchy sequence in the $b$-metric space $\left(X, d_{p_{b}}\right)$. Since $\left(X, p_{b}\right)$ is $p_{b}$-complete, then from Lemma $1,\left(X, d_{p_{b}}\right)$ is a $b$-complete $b$-metric space. Therefore, the sequence $\left\{x_{n}\right\}$ converges to some $z \in X$, that is, $\lim _{n \rightarrow \infty} d_{p_{b}}\left(x_{n}, z\right)=0$. Again, from Lemma 1 ,

$$
\lim _{n \rightarrow \infty} p_{b}\left(z, x_{n}\right)=\lim _{n \rightarrow \infty} p_{b}\left(x_{n}, x_{n}\right)=p_{b}(z, z)
$$

On the other hand, thanks to (3.6) and condition $\left(\mathrm{p}_{b 2}\right), \lim _{n \rightarrow \infty} p_{b}\left(x_{n}, x_{n}\right)=0$, which yields that

$$
\lim _{n \rightarrow \infty} p_{b}\left(z, x_{n}\right)=\lim _{n \rightarrow \infty} p_{b}\left(x_{n}, x_{n}\right)=p_{b}(z, z)=0 .
$$

Using the triangular inequality, we get

$$
p_{b}(z, f z) \leq s p_{b}\left(z, f x_{n}\right)+s p_{b}\left(f x_{n}, f z\right) .
$$

Letting $n \rightarrow \infty$ and using the continuity of $f$, we get

$$
p_{b}(z, f z) \leq s \lim _{n \rightarrow \infty} p_{b}\left(z, f x_{n}\right)+s \lim _{n \rightarrow \infty} p_{b}\left(f x_{n}, f z\right)=s p_{b}(f z, f z) .
$$

Note that from (3.1), we have

$$
\psi\left(s p_{b}(f z, f z)\right) \leq \psi\left(M_{s}^{f}(z, z)\right)-\varphi\left(M_{s}^{f}(z, z)\right)
$$


where

$$
M_{s}^{f}(z, z)=\max \left\{p_{b}(z, z), p_{b}(z, f z), p_{b}(z, f z), \frac{p_{b}(z, f z)+p_{b}(z, f z)}{2 s}\right\}=p_{b}(f z, z) .
$$

Hence, as $\psi$ is nondecreasing, we have $\operatorname{sp}_{b}(f z, f z) \leq p_{b}(f z, z)$. Thus, by (3.18) we obtain that $s p_{b}(f z, f z)=p_{b}(f z, z)$. But then, using (3.19), we get that $\varphi\left(M_{s}^{f}(z, z)\right)=0$.

Hence, we have $p_{b}(f z, z)=0$ and $f z=z$. Thus, $z$ is a fixed point of $f$.

We will show now that the continuity of $f$ in Theorem 1 is not necessary and can be replaced by another assumption.

Theorem 2 Under the hypotheses of Theorem 1, without the continuity assumption on $f$, assume that whenever $\left\{x_{n}\right\}$ is a nondecreasing sequence in $X$ such that $x_{n} \rightarrow x \in X$, one has $x_{n} \preceq x$ for all $n \in \mathbb{N}$. Then $f$ has a fixed point in $X$.

Proof Following similar arguments as those given in Theorem 1, we construct an increasing sequence $\left\{x_{n}\right\}$ in $X$ such that $x_{n} \rightarrow z$ for some $z \in X$. Using the assumption on $X$, we have $x_{n} \preceq z$ for all $n \in \mathbb{N}$. Now, we show that $f z=z$. By (3.1), we have

$$
\begin{aligned}
\psi\left(s p_{b}\left(x_{n+1}, f z\right)\right) & =\psi\left(s p_{b}\left(f x_{n}, f z\right)\right) \\
& \leq \psi\left(M_{s}^{f}\left(x_{n}, z\right)\right)-\varphi\left(M_{s}^{f}\left(x_{n}, z\right)\right),
\end{aligned}
$$

where

$$
\begin{aligned}
M_{s}^{f}\left(x_{n}, z\right) & =\max \left\{p_{b}\left(x_{n}, z\right), p_{b}\left(x_{n}, f x_{n}\right), p_{b}(z, f z), \frac{p_{b}\left(x_{n}, f z\right)+p_{b}\left(f x_{n}, z\right)}{2 s}\right\} \\
& =\max \left\{p_{b}\left(x_{n}, z\right), p_{b}\left(x_{n}, x_{n+1}\right), p_{b}(z, f z), \frac{p_{b}\left(x_{n}, f z\right)+p_{b}\left(x_{n+1}, z\right)}{2 s}\right\} .
\end{aligned}
$$

Letting $n \rightarrow \infty$ in (3.21) and using Lemma 2, we get

$$
\begin{aligned}
\frac{p_{b}(z, f z)}{2 s^{2}} & =\min \left\{p_{b}(z, f z), \frac{\frac{p_{b}(z, f z)}{s}}{2 s}\right\} \leq \liminf _{i \rightarrow \infty} M_{s}^{f}\left(x_{n}, z\right) \leq \limsup _{i \rightarrow \infty} M_{s}^{f}\left(x_{n}, z\right) \\
& \leq \max \left\{p_{b}(z, f z), \frac{s p_{b}(z, f z)}{2 s}\right\}=p_{b}(z, f z) .
\end{aligned}
$$

Again, taking the upper limit as $n \rightarrow \infty$ in (3.20) and using Lemma 2 and (3.22), we get

$$
\begin{aligned}
\psi\left(p_{b}(z, f z)\right) & =\psi\left(s-p_{b}(z, f z)\right) \leq \psi\left(s \limsup _{n \rightarrow \infty} p_{b}\left(x_{n+1}, f z\right)\right) \\
& \leq \psi\left(\limsup _{n \rightarrow \infty} M_{s}^{f}\left(x_{n}, z\right)\right)-\liminf _{n \rightarrow \infty} \varphi\left(M_{s}^{f}\left(x_{n}, z\right)\right) \\
& \leq \psi\left(p_{b}(z, f z)\right)-\varphi\left(\liminf _{n \rightarrow \infty} M_{s}^{f}\left(x_{n}, z\right)\right) .
\end{aligned}
$$

Therefore, $\varphi\left(\liminf _{n \rightarrow \infty} M_{s}^{f}\left(x_{n}, z\right)\right) \leq 0$, equivalently, $\liminf _{n \rightarrow \infty} M_{s}^{f}\left(x_{n}, z\right)=0$. Thus, from (3.22) we get $z=f z$, and hence $z$ is a fixed point of $f$. 
Corollary 1 Let $\left(X, \preceq, p_{b}\right)$ be a $p_{b}$-complete ordered partial b-metric space. Let $f: X \rightarrow X$ be a continuous mapping, nondecreasing with respect to $\preceq$. Suppose that there exists $k \in$ $[0,1)$ such that

$$
p_{b}(f x, f y) \leq \frac{k}{s} \max \left\{p_{b}(x, y), p_{b}(x, f x), p_{b}(y, f y), \frac{p_{b}(x, f y)+p_{b}(y, f x)}{2 s}\right\}
$$

for all comparable elements $x, y \in X$. If there exists $x_{0} \in X$ such that $x_{0} \preceq f x_{0}$, then $f$ has a fixed point.

Proof Follows from Theorem 1 by taking $\psi(t)=t$ and $\varphi(t)=(1-k) t$, for all $t \in[0,+\infty)$.

Corollary 2 Under the hypotheses of Corollary 1 , without the continuity assumption on $f$, for any nondecreasing sequence $\left\{x_{n}\right\}$ in $X$ such that $x_{n} \rightarrow x \in X$, let us have $x_{n} \preceq x$ for all $n \in \mathbb{N}$. Then $f$ has a fixed point in $X$.

Now, in order to support the usability of our results, we present the following example.

Example 4 Let $X=[0,+\infty)$ be equipped with the partial order $\preceq$ defined by

$$
x \preceq y \quad \Longleftrightarrow \quad x=y \vee(x, y \in[0,1] \wedge x \leq y),
$$

and with the partial $b$-metric $p_{b}$ given by $p_{b}(x, y)=[\max \{x, y\}]^{2}($ with $s=2)$. Consider the mapping $f: X \rightarrow X$ given by

$$
f x= \begin{cases}\frac{x}{\sqrt{2} \sqrt{1+x}}, & x \in[0,1] \\ \frac{x}{2}, & x>1 .\end{cases}
$$

Then $f$ is continuous and increasing, and $0 \preceq f 0$. Take altering distance functions

$$
\psi(t)=t, \quad \varphi(t)= \begin{cases}\frac{t \sqrt{t}}{1+\sqrt{t}}, & 0 \leq t \leq 1, \\ \frac{t}{2}, & t>1 .\end{cases}
$$

In order to check the contractive condition (3.1) of Theorem 1, without loss of generality, we may take $x, y \in X$ such that $y \preceq x$. Consider the following two possible cases.

Case $1.0 \leq y \leq x \leq 1$. Then

$$
p_{b}(f x, f y)=\left[\max \left\{\frac{x}{\sqrt{2} \sqrt{1+x}}, \frac{y}{\sqrt{2} \sqrt{1+y}}\right\}\right]^{2}=\frac{x^{2}}{2(1+x)}
$$

and

$$
M_{s}^{f}(x, y)=\max \left\{x^{2}, x^{2}, y^{2}, \frac{x^{2}+\max ^{2}\left\{y, \frac{x}{\sqrt{2} \sqrt{1+x}}\right\}}{2 s}\right\}=x^{2} .
$$

Thus, (3.1) reduces to

$$
\psi\left(2 \cdot \frac{x^{2}}{2(1+x)}\right)=\frac{x^{2}}{1+x} \leq x^{2}-\frac{x^{3}}{1+x}=\psi\left(x^{2}\right)-\varphi\left(x^{2}\right) .
$$


Case 2. $x=y \geq 1$. Then $p_{b}(f x, f y)=\frac{x^{2}}{4}$ and $M_{s}^{f}(x, y)=x^{2}$, so (3.1) reduces to

$$
\psi\left(2 \cdot \frac{x^{2}}{4}\right)=\frac{x^{2}}{2} \leq x^{2}-\frac{x^{2}}{2}=\psi\left(x^{2}\right)-\varphi\left(x^{2}\right) .
$$

Hence, all the conditions of Theorem 1 are satisfied and $f$ has a fixed point (which is $z=0$ ).

\section{Common fixed point results in partial $b$-metric spaces}

Let $\left(X, \preceq, p_{b}\right)$ be an ordered partial $b$-metric space with the coefficient $s \geq 1$, and let $f, g$ : $X \rightarrow X$ be two mappings. Set

$$
M_{s}^{f, g}(x, y)=\max \left\{p_{b}(x, y), p_{b}(x, f x), p_{b}(y, g y), \frac{p_{b}(x, g y)+p_{b}(y, f x)}{2 s}\right\}
$$

Now, we present the following definition.

Definition 12 Let $\left(X, \preceq, p_{b}\right)$ be an ordered partial $b$-metric space, and let $\psi$ and $\varphi$ be altering distance functions. We say that a pair $(f, g)$ of self-mappings $f, g: X \rightarrow X$ is a generalized $(\psi, \varphi)_{s}$-contraction pair if

$$
\psi\left(s^{2} p_{b}(f x, g y)\right) \leq \psi\left(M_{s}^{f, g}(x, y)\right)-\varphi\left(M_{s}^{f, g}(x, y)\right)
$$

for all comparable $x, y \in X$.

Definition 13 [42] Let $(X, \preceq)$ be a partially ordered set. Then two mappings $f, g: X \rightarrow X$ are said to be weakly increasing if $f x \preceq g f x$ and $g x \preceq f g x$ for all $x \in X$.

Theorem 3 Let $\left(X, \preceq, p_{b}\right)$ be a $p_{b}$-complete ordered partial b-metric space with the coefficient $s \geq 1$, and let $f, g: X \rightarrow X$ be two weakly increasing mappings with respect to $\preceq$. Suppose that $(f, g)$ is a generalized $(\psi, \varphi)_{s}$-contraction pair for some altering distance functions $\psi$ and $\varphi$. If $f$ and $g$ are continuous, then $f$ and $g$ have a common fixed point.

Proof Let us divide the proof into two parts as follows.

First part. We prove that $u \in X$ is a fixed point of $f$ if and only if it is a fixed point of $g$. Suppose that $u$ is a fixed point of $f$, that is, $f u=u$. As $u \preceq u$, by (4.1), we have

$$
\begin{aligned}
\psi( & \left.s^{2} p_{b}(u, g u)\right)=\psi\left(s^{2} p_{b}(f u, g u)\right) \\
\leq & \psi\left(\max \left\{p_{b}(u, u), p_{b}(u, f u), p_{b}(u, g u), \frac{1}{2 s}\left(p_{b}(u, g u)+p_{b}(u, f u)\right)\right\}\right) \\
& -\varphi\left(\max \left\{p_{b}(u, u), p_{b}(u, f u), p_{b}(u, g u), \frac{1}{2 s}\left(p_{b}(u, g u)+p_{b}(u, f u)\right)\right\}\right) \\
\leq & \psi\left(p_{b}(u, g u)\right)-\varphi\left(\max \left\{p_{b}(u, u), p_{b}(u, f u), p_{b}(u, g u), \frac{1}{2 s}\left(p_{b}(u, g u)+p_{b}(u, f u)\right)\right\}\right) \\
\leq & \psi\left(s^{2} p_{b}(u, g u)\right) \\
& -\varphi\left(\max \left\{p_{b}(u, u), p_{b}(u, f u), p_{b}(u, g u), \frac{1}{2 s}\left(p_{b}(u, g u)+p_{b}(u, f u)\right)\right\}\right) .
\end{aligned}
$$


Therefore, $p_{b}(u, g u)=0$ and hence $g u=u$. Similarly, we can show that if $u$ is a fixed point of $g$, then $u$ is a fixed point of $f$.

Second part (construction of a sequence by iterative technique).

Let $x_{0} \in X$. We construct a sequence $\left\{x_{n}\right\}$ in $X$ such that $x_{2 n+1}=f x_{2 n}$ and $x_{2 n+2}=g x_{2 n+1}$ for all nonnegative integers $n$. As $f$ and $g$ are weakly increasing with respect to $\preceq$, we have

$$
\begin{gathered}
x_{1}=f x_{0} \preceq g f x_{0}=x_{2}=g x_{1} \preceq f g x_{1}=x_{3} \preceq \cdots \\
\preceq x_{2 n+1}=f x_{2 n} \preceq g f x_{2 n}=x_{2 n+2} \preceq \cdots .
\end{gathered}
$$

If $x_{2 n}=x_{2 n+1}$ for some $n \in \mathbb{N}$, then $x_{2 n}=f x_{2 n}$. Thus $x_{2 n}$ is a fixed point of $f$. By the first part, we conclude that $x_{2 n}$ is also a fixed point of $g$.

If $x_{2 n+1}=x_{2 n+2}$ for some $n \in \mathbb{N}$, then $x_{2 n+1}=g x_{2 n+1}$. Thus, $x_{2 n+1}$ is a fixed point of $g$. By the first part, we conclude that $x_{2 n+1}$ is also a fixed point of $f$. Therefore, we assume that $x_{n} \neq x_{n+1}$ for all $n \in \mathbb{N}$. Now, we complete the proof in the following steps.

Step 1: We will prove that

$$
\lim _{n \rightarrow \infty} p_{b}\left(x_{n}, x_{n+1}\right)=0
$$

As $x_{2 n+1}$ and $x_{2 n+2}$ are comparable, by (4.1), we have

$$
\begin{aligned}
\psi\left(p_{b}\left(x_{2 n+1}, x_{2 n+2}\right)\right) & \leq \psi\left(s^{2} p_{b}\left(x_{2 n+1}, x_{2 n+2}\right)\right) \\
& =\psi\left(s^{2} p_{b}\left(f x_{2 n}, g x_{2 n+1}\right)\right) \\
& \leq \psi\left(M_{s}^{f, g}\left(x_{2 n}, x_{2 n+1}\right)\right)-\varphi\left(M_{s}^{f, g}\left(x_{2 n}, x_{2 n+1}\right)\right),
\end{aligned}
$$

where

$$
\begin{aligned}
M_{s}^{f, g}\left(x_{2 n}, x_{2 n+1}\right)= & \max \left\{p_{b}\left(x_{2 n}, x_{2 n+1}\right), p_{b}\left(x_{2 n}, f x_{2 n}\right), p_{b}\left(x_{2 n+1}, g x_{2 n+1}\right),\right. \\
& \left.\frac{p_{b}\left(f x_{2 n}, x_{2 n+1}\right)+p_{b}\left(x_{2 n}, g x_{2 n+1}\right)}{2 s}\right\} \\
= & \max \left\{p_{b}\left(x_{2 n}, x_{2 n+1}\right), p_{b}\left(x_{2 n+1}, x_{2 n+2}\right)\right. \\
& \left.\frac{p_{b}\left(x_{2 n+1}, x_{2 n+1}\right)+p_{b}\left(x_{2 n}, x_{2 n+2}\right)}{2 s}\right\} \\
\leq & \max \left\{p_{b}\left(x_{2 n}, x_{2 n+1}\right), p_{b}\left(x_{2 n+1}, x_{2 n+2}\right)\right. \\
& \left.\frac{s p_{b}\left(x_{2 n}, x_{2 n+1}\right)+s p_{b}\left(x_{2 n+1}, x_{2 n+2}\right)}{2 s}\right\} \\
= & \max \left\{p_{b}\left(x_{2 n}, x_{2 n+1}\right), p_{b}\left(x_{2 n+1}, x_{2 n+2}\right)\right\}
\end{aligned}
$$

Hence, we have

$$
\begin{aligned}
\psi\left(p_{b}\left(x_{2 n+1}, x_{2 n+2}\right)\right) \leq & \psi\left(\max \left\{p_{b}\left(x_{2 n}, x_{2 n+1}\right), p_{b}\left(x_{2 n+1}, x_{2 n+2}\right)\right\}\right) \\
& -\varphi\left(\max \left\{p_{b}\left(x_{2 n}, x_{2 n+1}\right), p_{b}\left(x_{2 n+1}, x_{2 n+2}\right)\right\}\right) .
\end{aligned}
$$


If

$$
\max \left\{p_{b}\left(x_{2 n}, x_{2 n+1}\right), p_{b}\left(x_{2 n+1}, x_{2 n+2}\right)\right\}=p_{b}\left(x_{2 n+1}, x_{2 n+2}\right),
$$

then (4.2) becomes

$$
\begin{aligned}
\psi\left(p_{b}\left(x_{2 n+1}, x_{2 n+2}\right)\right) & \leq \psi\left(p_{b}\left(x_{2 n+1}, x_{2 n+2}\right)\right)-\varphi\left(p_{b}\left(x_{2 n+1}, x_{2 n+2}\right)\right) \\
& <\psi\left(p_{b}\left(x_{2 n+1}, x_{2 n+2}\right)\right)
\end{aligned}
$$

which gives a contradiction. Hence,

$$
\max \left\{p_{b}\left(x_{2 n}, x_{2 n+1}\right), p_{b}\left(x_{2 n+1}, x_{2 n+2}\right)\right\}=p_{b}\left(x_{2 n}, x_{2 n+1}\right),
$$

and (4.2) becomes

$$
\begin{aligned}
\psi\left(p_{b}\left(x_{2 n+1}, x_{2 n+2}\right)\right) & \leq \psi\left(p_{b}\left(x_{2 n}, x_{2 n+1}\right)\right)-\varphi\left(p_{b}\left(x_{2 n}, x_{2 n+1}\right)\right) \\
& \leq \psi\left(p_{b}\left(x_{2 n}, x_{2 n+1}\right)\right)
\end{aligned}
$$

Similarly, we can show that

$$
\psi\left(p_{b}\left(x_{2 n+1}, x_{2 n}\right)\right) \leq \psi\left(p_{b}\left(x_{2 n-1}, x_{2 n}\right)\right)-\varphi\left(p_{b}\left(x_{2 n-1}, x_{2 n}\right)\right) \leq \psi\left(p_{b}\left(x_{2 n-1}, x_{2 n}\right)\right) .
$$

By (4.3) and (4.4), we get that $\left\{p_{b}\left(x_{n}, x_{n+1}\right): n \in \mathbb{N}\right\}$ is a nonincreasing sequence of positive numbers. Hence, there is $r \geq 0$ such that

$$
\lim _{n \rightarrow \infty} p_{b}\left(x_{n}, x_{n+1}\right)=r
$$

Letting $n \rightarrow \infty$ in (4.3), we get

$$
\psi(r) \leq \psi(r)-\varphi(r) \leq \psi(r)
$$

which implies that $\varphi(r)=0$ and hence $r=0$. So, we have

$$
\lim _{n \rightarrow \infty} p_{b}\left(x_{n}, x_{n}\right) \leq \lim _{n \rightarrow \infty} p_{b}\left(x_{n}, x_{n+1}\right)=0 .
$$

Step 2. We will prove that $\left\{x_{n}\right\}$ is a $p_{b}$-Cauchy sequence. Because of (4.5), it is sufficient to show that $\left\{x_{2 n}\right\}$ is a $p_{b}$-Cauchy sequence. By Lemma 1 , we should show that $\left\{x_{2 n}\right\}$ is $b$-Cauchy in $\left(X, d_{p_{b}}\right)$. Suppose the contrary, i.e., that $\left\{x_{2 n}\right\}$ is not a $b$-Cauchy sequence in $\left(X, d_{p_{b}}\right)$. Then there exists $\varepsilon>0$ for which we can find two subsequences $\left\{x_{2 m_{i}}\right\}$ and $\left\{x_{2 n_{i}}\right\}$ of $\left\{x_{2 n}\right\}$ such that $n_{i}$ is the smallest index for which

$$
n_{i}>m_{i}>i, \quad d_{p_{b}}\left(x_{2 m_{i}}, x_{2 n_{i}}\right) \geq \varepsilon
$$

This means that

$$
d_{p_{b}}\left(x_{2 m_{i}}, x_{2 n_{i}-2}\right)<\varepsilon \text {. }
$$


From (4.6) and using the triangular inequality, we get

$$
\varepsilon \leq d_{p_{b}}\left(x_{2 m_{i}}, x_{2 n_{i}}\right) \leq s d_{p_{b}}\left(x_{2 m_{i}}, x_{2 m_{i}+1}\right)+s d_{p_{b}}\left(x_{2 m_{i}+1}, x_{2 n_{i}}\right) .
$$

Using (4.5) and taking the upper limit as $i \rightarrow \infty$, we get

$$
\frac{\varepsilon}{s} \leq \limsup _{i \rightarrow \infty} d_{p_{b}}\left(x_{2 m_{i}+1}, x_{2 n_{i}}\right)
$$

On the other hand, we have

$$
d_{p_{b}}\left(x_{2 m_{i}}, x_{2 n_{i}-1}\right) \leq s d_{p_{b}}\left(x_{2 m_{i}}, x_{2 n_{i}-2}\right)+s d_{p_{b}}\left(x_{2 n_{i}-2}, x_{2 n_{i}-1}\right) .
$$

Using (4.5), (4.7) and taking the upper limit as $i \rightarrow \infty$, we get

$$
\limsup _{i \rightarrow \infty} d_{p_{b}}\left(x_{2 m_{i}}, x_{2 n_{i}-1}\right) \leq \varepsilon s
$$

Again, using the triangular inequality, we have

$$
\begin{aligned}
d_{p_{b}}\left(x_{2 m_{i}}, x_{2 n_{i}}\right) & \leq s d_{p_{b}}\left(x_{2 m_{i}}, x_{2 n_{i}-2}\right)+s d_{p_{b}}\left(x_{2 n_{i}-2}, x_{2 n_{i}}\right) \\
& \leq s d_{p_{b}}\left(x_{2 m_{i}}, x_{2 n_{i}-2}\right)+s^{2} d_{p_{b}}\left(x_{2 n_{i}-2}, x_{2 n_{i}-1}\right)+s^{2} d_{p_{b}}\left(x_{2 n_{i}-1}, x_{2 n_{i}}\right)
\end{aligned}
$$

and

$$
d_{p_{b}}\left(x_{2 m_{i}+1}, x_{2 n_{i}-1}\right) \leq s d_{p_{b}}\left(x_{2 m_{i}+1}, x_{2 m_{i}}\right)+s d_{p_{b}}\left(x_{2 m_{i}}, x_{2 n_{i}-1}\right)
$$

Taking the upper limit as $i \rightarrow \infty$ in the above inequalities and using (4.5), (4.7) and (4.8), we get

$$
\limsup _{i \rightarrow \infty} d_{p_{b}}\left(x_{2 m_{i}}, x_{2 n_{i}}\right) \leq \varepsilon s
$$

and

$$
\limsup _{i \rightarrow \infty} d_{p_{b}}\left(x_{2 m_{i}+1}, x_{2 n_{i}-1}\right) \leq \varepsilon s^{2}
$$

From the definition of $d_{p_{b}}$ and (4.5), we have the following relations:

$$
\begin{aligned}
& \frac{\varepsilon}{2 s} \leq \limsup _{i \rightarrow \infty} p_{b}\left(x_{2 m_{i}+1}, x_{2 n_{i}}\right), \\
& \frac{\varepsilon}{2 s} \leq \liminf _{i \rightarrow \infty} p_{b}\left(x_{2 m_{i}}, x_{2 n_{i}-1}\right) \leq \limsup _{i \rightarrow \infty} p_{b}\left(x_{2 m_{i}}, x_{2 n_{i}-1}\right) \leq \frac{s \varepsilon}{2}, \\
& \underset{i \rightarrow \infty}{\limsup _{i \rightarrow \infty} p_{b}\left(x_{2 m_{i}}, x_{2 n_{i}}\right) \leq \frac{s \varepsilon}{2},} \\
& \limsup _{i \rightarrow \infty} p_{b}\left(x_{2 m_{i}+1}, x_{2 n_{i}-1}\right) \leq \frac{s^{2} \varepsilon}{2} .
\end{aligned}
$$


Since $x_{2 m_{i}}$ and $x_{2 n_{i}-1}$ are comparable, using (4.1) we have

$$
\begin{aligned}
\psi\left(s^{2} p_{b}\left(x_{2 m_{i}+1}, x_{2 n_{i}}\right)\right) & =\psi\left(s^{2} p_{b}\left(f x_{2 m_{i}}, g x_{2 n_{i}-1}\right)\right) \\
& \leq \psi\left(M_{s}^{f, g}\left(x_{2 m_{i}}, x_{2 n_{i}-1}\right)\right)-\varphi\left(M_{s}^{f, g}\left(x_{2 m_{i}}, x_{2 n_{i}-1}\right)\right)
\end{aligned}
$$

where

$$
\begin{aligned}
M_{s}^{f, g}\left(x_{2 m_{i}}, x_{2 n_{i}-1}\right)= & \max \left\{p_{b}\left(x_{2 m_{i}}, x_{2 n_{i}-1}\right), p_{b}\left(x_{2 m_{i}}, x_{2 m_{i}+1}\right), p_{b}\left(x_{2 n_{i}-1}, x_{2 n_{i}}\right),\right. \\
& \left.\frac{p_{b}\left(x_{2 m_{i}}, x_{2 n_{i}}\right)+p_{b}\left(x_{2 m_{i}+1}, x_{2 n_{i}-1}\right)}{2 s}\right\}
\end{aligned}
$$

Taking the upper limit in (4.14) and using (4.5) and (4.10)-(4.12), we get

$$
\begin{aligned}
\limsup _{i \rightarrow \infty} M_{s}^{f, g}\left(x_{2 m_{i}}, x_{2 n_{i}-1}\right)= & \max \left\{\limsup _{i \rightarrow \infty} p_{b}\left(x_{2 m_{i}}, x_{2 n_{i}-1}\right), 0,0,\right. \\
& \left.\frac{\limsup _{i \rightarrow \infty} p_{b}\left(x_{2 m_{i}}, x_{2 n_{i}}\right)+\lim \sup _{i \rightarrow \infty} p_{b}\left(x_{2 m_{i}+1}, x_{2 n_{i}-1}\right)}{2 s}\right\} \\
\leq & \max \left\{\frac{s \varepsilon}{2}, \frac{\frac{\varepsilon s+\varepsilon s^{2}}{2}}{2 s}\right\}=\frac{s \varepsilon}{2} .
\end{aligned}
$$

Now, taking the upper limit as $i \rightarrow \infty$ in (4.13) and using (4.9) and (4.15), we have

$$
\begin{aligned}
\psi\left(\frac{s \varepsilon}{2}\right) & =\psi\left(s^{2} \frac{\varepsilon}{2 s}\right) \leq \psi\left(s^{2} \limsup _{i \rightarrow \infty} p_{b}\left(x_{2 m_{i}+1}, x_{2 n_{i}}\right)\right) \\
& \leq \psi\left(\limsup _{i \rightarrow \infty}^{f, g} M_{s}^{f,}\left(x_{2 m_{i}}, x_{2 n_{i}-1}\right)\right)-\varphi\left(\liminf _{i \rightarrow \infty} M_{s}^{f, g}\left(x_{2 m_{i}}, x_{2 n_{i}-1}\right)\right) \\
& \leq \psi\left(\frac{s \varepsilon}{2}\right)-\varphi\left(\liminf _{i \rightarrow \infty} M_{s}^{f, g}\left(x_{2 m_{i}}, x_{2 n_{i}-1}\right)\right)
\end{aligned}
$$

which implies that $\varphi\left(\liminf _{i \rightarrow \infty} M_{s}^{f, g}\left(x_{2 m_{i}}, x_{2 n_{i}-1}\right)\right)=0$. By (4.14), it follows that

$$
\liminf _{i \rightarrow \infty} p_{b}\left(x_{2 m_{i}}, x_{2 n_{i}-1}\right)=0
$$

which is in contradiction with (4.10). Thus, we have proved that $\left\{x_{n}\right\}$ is a $b$-Cauchy sequence in the metric space $\left(X, d_{p_{b}}\right)$. Since $\left(X, p_{b}\right)$ is $p_{b}$-complete, then from Lemma 1 , $\left(X, d_{p_{b}}\right)$ is a $b$-complete $b$-metric space. Therefore, the sequence $\left\{x_{n}\right\}$ converges to some $z \in X$, that is, $\lim _{n \rightarrow \infty} d_{p_{b}}\left(x_{n}, z\right)=0$. Again, from Lemma 1 ,

$$
\lim _{n \rightarrow \infty} p_{b}\left(z, x_{n}\right)=\lim _{n \rightarrow \infty} p_{b}\left(x_{n}, x_{n}\right)=p_{b}(z, z)
$$

On the other hand, from (4.5) we get that

$$
\lim _{n \rightarrow \infty} p_{b}\left(z, x_{n}\right)=\lim _{n \rightarrow \infty} p_{b}\left(x_{n}, x_{n}\right)=p_{b}(z, z)=0 .
$$


Step 3 (Existence of a common fixed point). Using the triangular inequality, we get

$$
\begin{aligned}
& p_{b}(z, f z) \leq s p_{b}\left(z, f x_{2 n}\right)+s p_{b}\left(f x_{2 n}, f z\right), \\
& p_{b}(z, g z) \leq s p_{b}\left(z, g x_{2 n+1}\right)+s p_{b}\left(g x_{2 n+1}, g z\right) .
\end{aligned}
$$

Letting $n \rightarrow \infty$ and using the continuity of $f$ and $g$, we get

$$
\begin{aligned}
& p_{b}(z, f z) \leq s \lim _{n \rightarrow \infty} p_{b}\left(z, f x_{2 n}\right)+s \lim _{n \rightarrow \infty} p_{b}\left(f x_{2 n}, f z\right)=s p_{b}(f z, f z), \\
& p_{b}(z, g z) \leq s \lim _{n \rightarrow \infty} p_{b}\left(z, g x_{2 n+1}\right)+s \lim _{n \rightarrow \infty} p_{b}\left(g x_{2 n+1}, g z\right)=s p_{b}(g z, g z) .
\end{aligned}
$$

Therefore,

$$
\max \left\{p_{b}(z, f z), p_{b}(z, g z)\right\} \leq \max \left\{s p_{b}(f z, f z), s p_{b}(g z, g z)\right\} \leq s^{2} p_{b}(g z, f z) .
$$

From (4.1), we have

$$
\psi\left(s^{2} p_{b}(f z, g z)\right) \leq \psi\left(M_{s}^{f, g}(z, z)\right)-\varphi\left(M_{s}^{f, g}(z, z)\right)
$$

where

$$
\begin{aligned}
M_{s}^{f, g}(z, z) & =\max \left\{p_{b}(z, z), p_{b}(z, f z), p_{b}(z, g z), \frac{p_{b}(z, g z)+p_{b}(z, f z)}{2 s}\right\} \\
& =\max \left\{p_{b}(z, f z), p_{b}(z, g z)\right\} .
\end{aligned}
$$

As $\psi$ is nondecreasing, we have $s^{2} p_{b}(f z, g z) \leq \max \left\{p_{b}(z, f z), p_{b}(z, g z)\right\}$. Hence, by (4.16) we obtain that $s^{2} p_{b}(f z, g z)=\max \left\{p_{b}(z, f z), p_{b}(z, g z)\right\}$. But then, using (4.17), we get that $\varphi\left(M_{s}^{f, g}(z, z)\right)=0$. Thus, we have $f z=g z=z$ and $z$ is a common fixed point of $f$ and $g$.

The continuity of functions $f$ and $g$ in Theorem 3 can be replaced by another condition.

Theorem 4 Under the hypotheses of Theorem 3, without the continuity assumption on the functions $f$ and $g$, for any nondecreasing sequence $\left\{x_{n}\right\}$ in $X$ such that $x_{n} \rightarrow x \in X$, let us have $x_{n} \preceq x$ for all $n \in \mathbb{N}$. Then $f$ and $g$ have a common fixed point in $X$.

Proof Reviewing the proof of Theorem 3, we construct an increasing sequence $\left\{x_{n}\right\}$ in $X$ such that $x_{n} \rightarrow z$ for some $z \in X$. Using the given assumption on $X$, we have $x_{n} \preceq z$ for all $n \in \mathbb{N}$. Now, we show that $f z=g z=z$. By (4.1), we have

$$
\begin{aligned}
\psi\left(s^{2} p_{b}\left(x_{2 n+1}, g z\right)\right) & =\psi\left(s^{2} p_{b}\left(f x_{2 n}, g z\right)\right) \\
& \leq \psi\left(M_{s}^{f, g}\left(x_{2 n}, z\right)\right)-\varphi\left(M_{s}^{f, g}\left(x_{2 n}, z\right)\right),
\end{aligned}
$$

where

$$
\begin{aligned}
M_{s}^{f, g}\left(x_{2 n}, z\right) & =\max \left\{p_{b}\left(x_{2 n}, z\right), p_{b}\left(x_{2 n}, f x_{2 n}\right), p_{b}(z, g z), \frac{p_{b}\left(x_{2 n}, g z\right)+p_{b}\left(f x_{2 n}, z\right)}{2 s}\right\} \\
& =\max \left\{p_{b}\left(x_{2 n}, z\right), p_{b}\left(x_{2 n}, x_{2 n+1}\right), p_{b}(z, g z), \frac{p_{b}\left(x_{2 n}, g z\right)+p_{b}\left(x_{2 n+1}, z\right)}{2 s}\right\} .
\end{aligned}
$$


Letting $n \rightarrow \infty$ in (4.19) and using Lemma 2, we get

$$
\begin{aligned}
\frac{p_{b}(z, g z)}{s^{2}} & \leq \max \left\{p_{b}(z, g z), \frac{\frac{p_{b}(z, g z)}{s}}{2 s}\right\} \leq \liminf _{n \rightarrow \infty} M_{s}^{f, g}\left(x_{2 n}, z\right) \\
& \leq \limsup _{n \rightarrow \infty} M_{s}^{f, g}\left(x_{2 n}, z\right) \leq \max \left\{p_{b}(z, g z), \frac{s p_{b}(z, g z)}{2 s}\right\}=p_{b}(z, g z) .
\end{aligned}
$$

Again, taking the upper limit as $n \rightarrow \infty$ in (4.18) and using Lemma 2 and (4.20), we get

$$
\begin{aligned}
\psi\left(p_{b}(z, g z)\right) & =\psi\left(s^{2} \frac{1}{s^{2}} p_{b}(z, g z)\right) \leq \psi\left(s^{2} \limsup _{n \rightarrow \infty} d\left(x_{2 n+1}, g z\right)\right) \\
& \leq \psi\left(\limsup _{n \rightarrow \infty} M_{s}^{f, g}\left(x_{2 n}, z\right)\right)-\varphi\left(\liminf _{n \rightarrow \infty} M_{s}^{f, g}\left(x_{2 n}, z\right)\right) \\
& \leq \psi\left(p_{b}(z, g z)\right)-\varphi\left(\liminf _{n \rightarrow \infty} M_{s}^{f, g}\left(x_{2 n}, z\right)\right) .
\end{aligned}
$$

Therefore, $\varphi\left(\liminf _{n \rightarrow \infty} M_{s}^{f, g}\left(x_{2 n}, z\right)\right) \leq 0$, equivalently, $\liminf _{n \rightarrow \infty} M_{s}^{f, g}\left(x_{2 n}, z\right)=0$. Thus, from (4.20) we get $z=g z$ and hence $z$ is a fixed point of $g$. On the other hand, similar to the first part of the proof of Theorem 3, we can show that $f z=z$. Hence, $z$ is a common fixed point of $f$ and $g$.

Also, we have the following results.

Corollary 3 Let $\left(X, \preceq, p_{b}\right)$ be a $p_{b}$-complete ordered partial b-metric space with the coefficient $s \geq 1$, and let $f, g: X \rightarrow X$ be two weakly increasing mappings with respect to $\preceq$. Suppose that there exists $k \in[0,1)$ such that

$$
p_{b}(f x, g y) \leq \frac{k}{s^{2}} \max \left\{p_{b}(x, y), p_{b}(x, f x), p_{b}(y, g y), \frac{p_{b}(x, g y)+p_{b}(f x, y)}{2 s}\right\}
$$

for all comparable elements $x, y \in X$. Iff and $g$ are continuous, then $f$ and $g$ have a common fixed point.

Corollary 4 Under the hypotheses of Corollary 3, without the continuity assumption on the functions $f$ and $g$, assume that whenever $\left\{x_{n}\right\}$ is a nondecreasing sequence in $X$ such that $x_{n} \rightarrow x \in X$, then $x_{n} \preceq x$ for all $n \in \mathbb{N}$. Then $f$ and $g$ have a common fixed point in $X$.

Remark 1 Recall that a subset $W$ of a partially ordered set $X$ is said to be well ordered if every two elements of $W$ are comparable. Note that in Theorems 1 and 2, it can be proved in a standard way that $f$ has a unique fixed point provided that the fixed points of $f$ are comparable. Similarly, in Theorems 3 and 4, the set of common fixed points of $f$ and $g$ is well ordered if and only if $f$ and $g$ have one and only one common fixed point.

The usability of these results is demonstrated by the following example.

Example 5 Let $X=\{0,1,2,3,4\}$ be equipped with the following partial order $\preceq$ :

$$
\preceq:=\{(0,0),(1,1),(1,2),(2,2),(3,3),(4,2),(4,4)\} .
$$


Define a partial $b$-metric $p_{b}: X \times X \rightarrow \mathbb{R}^{+}$by

$$
p_{b}(x, y)= \begin{cases}0 & \text { if } x=y \\ (x+y)^{2} & \text { if } x \neq y\end{cases}
$$

It is easy to see that $\left(X, p_{b}\right)$ is a $p_{b}$-complete partial $b$-metric space, with $s=49 / 25$.

Define self-maps $f$ and $g$ by

$$
f=\left(\begin{array}{lllll}
0 & 1 & 2 & 3 & 4 \\
0 & 2 & 2 & 1 & 2
\end{array}\right), \quad g=\left(\begin{array}{lllll}
0 & 1 & 2 & 3 & 4 \\
0 & 2 & 2 & 1 & 1
\end{array}\right) \text {. }
$$

We see that $f$ and $g$ are weakly increasing mappings with respect to $\preceq$ and that $f$ and $g$ are continuous.

Define $\psi, \varphi:[0, \infty) \rightarrow[0, \infty)$ by $\psi(t)=\sqrt{t}$ and $\varphi(t)=\frac{t}{300}$. In order to check that $(f, g)$ is a generalized $(\psi, \varphi)_{s}$-contractive pair, only the case $x=2, y=4$ is nontrivial (when $x$ and $y$ are comparable and the left-hand side of condition (4.1) is positive). Then

$$
\psi\left(s^{2} p_{b}(f 2, g 4)\right)=\sqrt{s^{2} \cdot 3^{2}}=\frac{147}{25}=\sqrt{36}-\frac{36}{300}=\psi\left(M_{s}^{f, g}(2,4)\right)-\varphi\left(M_{s}^{f, g}(2,4)\right) .
$$

Thus, all the conditions of Theorem 3 are satisfied and hence $f$ and $g$ have common fixed points. Indeed, 0 and 2 are two common fixed points of $f$ and $g$. Note that the ordered set $(\{0,2\}, \preceq)$ is not well ordered.

Note that if the same example is considered in the space without order, then the contractive condition is not satisfied. For example,

$$
\begin{aligned}
\psi\left(s^{2} p_{b}(f 1, g 4)\right) & =\sqrt{s^{2} \cdot 3^{2}}=\frac{147}{25} \\
& >\frac{59}{12}=\sqrt{25}-\frac{25}{300}=\psi\left(M_{s}^{f, g}(1,4)\right)-\varphi\left(M_{s}^{f, g}(1,4)\right) .
\end{aligned}
$$

\section{Competing interests}

The authors declare that they have no competing interests.

\section{Authors' contributions}

All authors contributed equally and significantly in writing this paper. All authors read and approved the final manuscript.

\section{Author details}

${ }^{1}$ Department of Mathematics, The Hashemite University, P.O. 330127, Zarqa, 13115, Jordan. ${ }^{2}$ Department of Mathematics, Qaemshahr Branch, Islamic Azad University, Qaemshahr, Iran. ${ }^{3}$ Young Researchers and Elite Club, Kermanshah Branch, Islamic Azad University, Kermanshah, Iran. ${ }^{4}$ Faculty of Mathematics, University of Belgrade, Beograd, Serbia.

\section{Acknowledgements}

The fourth author is thankful to the Ministry of Education, Science and Technological Development of Serbia.

Received: 11 October 2013 Accepted: 31 October 2013 Published: 25 Nov 2013

\section{References}

1. Ran, ACM, Reurings, MCB: A fixed point theorem in partially ordered sets and some application to matrix equations. Proc. Am. Math. Soc. 132, 1435-1443 (2004)

2. Nieto, JJ, Lopez, RR: Contractive mapping theorems in partially ordered sets and applications to ordinary differential equations. Order 22, 223-239 (2005)

3. Abbas, M, Nazir, T, Radenović, S: Common fixed points of four maps in partially ordered metric spaces. Appl. Math. Lett. 24, 1520-1526 (2011) 
4. Aghajani, A, Radenović, S, Roshan, JR: Common fixed point results for four mappings satisfying almost generalized $(S, T)$-contractive condition in partially ordered metric spaces. Appl. Math. Comput. 218, 5665-5670 (2012)

5. Ćirić, Lj, Abbas, M, Saadati, R, Hussain, N: Common fixed points of almost generalized contractive mappings in ordered metric spaces. Appl. Math. Comput. 217, 5784-5789 (2011)

6. Radenović, S, Kadelburg, Z: Generalized weak contractions in partially ordered metric spaces. Comput. Math. Appl. 60, 1776-1783 (2010)

7. Radenović, S, Kadelburg, Z, Jandrlić, D, Jandrlić, A: Some results on weak contraction maps. Bull. Iran. Math. Soc. 38, 625-645 (2012)

8. Shatanawi, W, Mustafa, Z, Tahat, N: Some coincidence point theorems for nonlinear contractions in ordered metric spaces. Fixed Point Theory Appl. 2011, 68 (2011). doi:10.1186/1687-1812-2011-68

9. Bakhtin, IA: The contraction principle in quasimetric spaces. Funct. Anal. 30, 26-37 (1989)

10. Czerwik, S: Contraction mappings in b-metric spaces. Acta Math. Inform. Univ. Ostrav. 1, 5-11 (1993)

11. Czerwik, S: Nonlinear set-valued contraction mappings in b-metric spaces. Atti Semin. Mat. Fis. Univ. Modena 46, 263-276 (1998)

12. Aghajani, A, Abbas, M, Roshan, JR: Common fixed point of generalized weak contractive mappings in partially ordered $b$-metric spaces. Math. Slovaca (2012, in press)

13. Parvaneh, V, Roshan, JR, Radenović, S: Existence of tripled coincidence points in ordered $b$-metric spaces and an application to a system of integral equations. Fixed Point Theory Appl. 2013, 130 (2013). doi:10.1186/1687-1812-2013-130

14. Roshan, JR, Parvaneh, V, Sedghi, S, Shobkolaei, N, Shatanawi, W: Common fixed points of almost generalized $(\psi, \varphi)_{s}$-contractive mappings in ordered $b$-metric spaces. Fixed Point Theory Appl. 2013, 159 (2013). doi:10.1186/1687-1812-2013-159

15. Aydi, H, Bota, M-F, Karapinar, E, Moradi, S: A common fixed point for weak $\phi$-contractions on $b$-metric spaces. Fixed Point Theory 13(2), 337-346 (2012)

16. Aydi, H, Bota, M-F, Karapınar, E, Mitrović, S: A fixed point theorem for set-valued quasi-contractions in $b$-metric spaces. Fixed Point Theory Appl. 2012, 88 (2012). doi:10.1186/1687-1812-2012-88

17. Boriceanu, M: Fixed point theory for multivalued generalized contractions on a set with two $b$-metrics. Stud. Univ. Babeş-Bolyai, Math. LIV(3), 3-14 (2009)

18. Boriceanu, M: Strict fixed point theorems for multivalued operators in b-metric spaces. Int. J. Mod. Math. 4(3), 285-301 (2009)

19. Hussain, N, Đorić, D, Kadelburg, Z, Radenović, S: Suzuki-type fixed point results in metric type spaces. Fixed Point Theory Appl. 2012, 126 (2012). doi:10.1186/1687-1812-2012-126

20. Hussain, N, Shah, MH: KKM mappings in cone b-metric spaces. Comput. Math. Appl. 62, 1677-1684 (2011)

21. Khamsi, MA, Hussain, N: KKM mappings in metric type spaces. Nonlinear Anal. 73, 3123-3129 (2010)

22. Khamsi, MA: Remarks on cone metric spaces and fixed point theorems of contractive mappings. Fixed Point Theory Appl. 2010, Article ID 315398 (2010). doi:10.1155/2010/315398

23. Jovanović, M, Kadelburg, Z, Radenović, S: Common fixed point results in metric-type spaces. Fixed Point Theory Appl. 2010, Article ID 978121 (2010). doi:10.1155/2010/978121

24. Pacurar, M: Sequences of almost contractions and fixed points in $b$-metric spaces. An. Univ. Vest. Timiş., Ser. Mat.-Inform. 3, 125-137 (2010)

25. Roshan, JR, Shobkolaei, N, Sedghi, S, Abbas, M: Common fixed point of four maps in b-metric spaces. Hacet. J. Math. Stat. (2013, in press)

26. Singh, SL, Prasad, B: Some coincidence theorems and stability of iterative procedures. Comput. Math. Appl. 55, $2512-2520$ (2008)

27. Matthews, SG: Partial metric topology. Proc. 8th Summer Conference on General Topology and Applications. Ann. N.Y. Acad. Sci. 728, 183-197 (1994)

28. Aydi, H: Common fixed point results for mappings satisfying $(\psi, \varphi)$-weak contractions in ordered partial metric spaces. Int. J. Math. Stat. 12, 2 (2012)

29. Kadelburg, Z, Nashine, HK, Radenović, S: Fixed point results under various contractive conditions in partial metric spaces. Rev. R. Acad. Cienc. Exactas Fís. Nat., Ser. A Mat. 107, 241-256 (2013)

30. Nashine, HK, Kadelburg, Z, Radenović, S: Common fixed point theorems for weakly isotone increasing mappings in ordered partial metric spaces. Math. Comput. Model. 57, 2355-2365 (2013)

31. Oltra, S, Valero, O: Banach's fixed point theorem for partial metric spaces. Rend. Ist. Mat. Univ. Trieste 36, 17-26 (2004)

32. Sedghi, S, Shobkolaei, N: Common fixed point of maps in complete partial metric spaces. East Asian Math. J. 29, 1-12 (2013)

33. Shobkolaei, N, Sedghi, S, Roshan, JR, Altun, I: Common fixed point of mappings satisfying almost generalized $(S, T)$-contractive condition in partially ordered partial metric spaces. Appl. Math. Comput. 219, 443-452 (2012)

34. Shukla, S: Partial $b$-metric spaces and fixed point theorems. Mediterr. J. Math. (to appear). doi:10.1007/s00009-013-0327-4

35. Khan, MS, Swaleh, M, Sessa, S: Fixed point theorems by altering distances between the points. Bull. Aust. Math. Soc 30, 1-9 (1984)

36. Đorić, D: Common fixed point for generalized $(\psi, \varphi)$-weak contraction. Appl. Math. Lett. 22, 1896-1900 (2009)

37. Karapinar, E, Sadarangani, K: Fixed point theory for cyclic $(\psi, \varphi)$-contractions. Fixed Point Theory Appl. 2011, 69 (2011). doi:10.1186/1687-1812-2011-69

38. Nashine, $\mathrm{NH}$, Samet, B: Fixed point results for mappings satisfying $(\psi, \varphi)$-weakly contractive condition in partially ordered metric spaces. Nonlinear Anal. 74, 2201-2209 (2011)

39. Nashine, NH, Samet, B, Kim, J: Fixed point results for contractions involving generalized altering distances in ordered metric spaces. Fixed Point Theory Appl. 2011, 5 (2011). doi:10.1186/1687-1812-2011-5

40. Popescu, O: Fixed points for $(\psi, \varphi)$-weak contractions. Appl. Math. Lett. 24, 1-4 (2011)

41. Shatanawi, W, Samet, B: On $(\psi, \varphi)$-weakly contractive condition in partially ordered metric spaces. Comput. Math. Appl. 62, 3204-3214 (2011)

42. Altun, I, Damjanović, B, Đorić, D: Fixed point and common fixed point theorems on ordered cone metric spaces. Appl. Math. Lett. 23, 310-316 (2010) 
10.1186/1029-242X-2013-562

Cite this article as: Mustafa et al.: Some common fixed point results in ordered partial $b$-metric spaces. Journal of Inequalities and Applications 2013, 2013:562

Submit your manuscript to a SpringerOpen ${ }^{\circ}$ journal and benefit from:

- Convenient online submission

- Rigorous peer review

- Immediate publication on acceptance

Open access: articles freely available online

- High visibility within the field

- Retaining the copyright to your article

Submit your next manuscript at $\gg$ springeropen.com 